\title{
The Spinal Control of Backward Locomotion
}

\author{
Jonathan Harnie, ${ }^{1}$ Johannie Audet, ${ }^{2}$ Alexander N. Klishko, ${ }^{1}$ Adam Doelman, ${ }^{2}$ Boris I. Prilutsky, ${ }^{1}$ and \\ Alain Frigon ${ }^{1}$ \\ ${ }^{1}$ Department of Pharmacology-Physiology, Faculty of Medicine and Health Sciences, Université de Sherbrooke, Sherbrooke, Quebec J1H 5N4, \\ Canada, and ${ }^{2}$ School of Biological Sciences, Georgia Institute of Technology, Atlanta, Georgia 30332
}

Animal locomotion requires changing direction, from forward to backward. Here, we tested the hypothesis that sensorimotor circuits within the spinal cord generate backward locomotion and adjust it to task demands. We collected kinematic and electromyography (EMG) data during forward and backward locomotion at different treadmill speeds before and after complete spinal transection in six adult cats (three males and three females). After spinal transection, five/six cats performed backward locomotion, which required tonic somatosensory input in the form of perineal stimulation. One spinal cat performed forward locomotion but not backward locomotion while two others stepped backward but not forward. Spatiotemporal adjustments to increasing speed were similar in intact and spinal cats during backward locomotion and strategies were similar to forward locomotion, with shorter cycle and stance durations and longer stride lengths. Patterns of muscle activations, including muscle synergies, were similar for forward and backward locomotion in spinal cats. Indeed, we identified five muscle synergies that were similar during forward and backward locomotion. Lastly, spinal cats also stepped backward on a split-belt treadmill, with the left and right hindlimbs stepping at different speeds. Therefore, our results show that spinal sensorimotor circuits generate backward locomotion but require additional excitability compared with forward locomotion. Similar strategies for speed modulation and similar patterns of muscle activations and muscle synergies during forward and backward locomotion are consistent with a shared spinal locomotor network, with sensory feedback from the limbs controlling the direction.

Key words: central pattern generator; locomotor direction; muscle synergies; sensory feedback; spinal cord

Significance Statement

Animal locomotion requires changing direction, including forward, sideways and backward. This paper shows that the center controlling locomotion within the spinal cord can produce a backward pattern when instructed by sensory signals from the limbs. However, the spinal locomotor network requires greater excitability to produce backward locomotion compared with forward locomotion. The paper also shows that the spinal network controlling locomotion in the forward direction also controls locomotion in the backward direction.

\section{Introduction}

Locomotion in different directions is essential when adjusting to the environment and for goal-oriented behaviors. Although dynamic sensorimotor interactions between supraspinal inputs, spinal circuits and somatosensory feedback generate locomotion (Rossignol et al., 2006), their relative contributions in different directions is not well understood. A specialized network within

\footnotetext{
Received Apr. 8, 2020; revised Nov. 16, 2020; accepted Nov. 18, 2020.

B.I.P. and A.F. are equally contributing senior authors.

Acknowledgements: We thank Philippe Drapeau (Université de Montréal) from the Rossignol and Drew labs for developing the data collection and analysis software. This work was supported by the Canadian Institutes of Health Research Grant PJT-156296 and the Natural Sciences and Engineering Research Council of Canada Grant RGPIN-2016-03790 (to A.F.), by the National Institutes of Health Grant R01 NS110550 (to A.F. and B.I.P.), and by the National Institute of Neurological Disorders and Stroke Grant NS100928 (to B.I.P.).

Author contributions: J.H. and A.F. designed research; J.H., A.D., and A.F. performed research; J.H., J.A., A.N.K., and B.I.P. analyzed data; J.H., A.N.K., B.I.P., and A.F. wrote the paper.

The authors declare no competing financial interests.

Correspondence should be addressed to Alain Frigon at alain.frigon@usherbrooke.ca.

https://doi.org/10.1523/JNEUROSCI.0816-20.2020

Copyright $\odot 2021$ the authors
}

the spinal cord termed the locomotor central pattern generator (CPG) produces the basic pattern for forward locomotion (for review, see Grillner, 1981; McCrea and Rybak, 2008; Kiehn, 2011, 2016; Grillner and El Manira, 2015). In cats with a complete spinal transection (i.e., spinal cats), hindlimb locomotion recovers and adjusts to different treadmill speeds and to different speeds for the left and right hindlimbs on a split-belt treadmill (Forssberg et al., 1980; Frigon et al., 2013, 2017). This indicates that sensory feedback from the limbs adjusts the output of the spinal locomotor CPG for different forward speeds and directions.

What about locomotion in the backward direction? The neural control of backward locomotion has been investigated in intact or decerebrate cats (Buford and Smith, 1990, 1993; Buford et al., 1990; Perell et al., 1993; Pratt et al., 1996; Trank and Smith, 1996; Musienko et al., 2007, 2012; Zelenin et al., 2011; Merkulyeva et al., 2018; Deliagina et al., 2019) and humans (Thorstensson, 1986; Winter et al., 1989; Duysens et al., 1996; Grasso et al., 1998; Choi and Bastian, 2007; Ivanenko et al., 2008; 
Hoogkamer et al., 2014; Mahaki et al., 2017). Studies in cats and humans have shown that backward locomotor kinematics are mainly a reversal of forward locomotion (Thorstensson, 1986; Grasso et al., 1998; Winter et al., 1989; Buford et al., 1990; Viviani et al., 2011). While some have proposed that distinct functional networks generate forward and backward locomotion (Choi and Bastian, 2007; Mahaki et al., 2017), others have argued that they share common neural circuits (Grillner, 1981, 1985; Winter et al., 1989; Buford and Smith, 1990; Matsushima and Grillner, 1992; Duysens et al., 1996; Lamb and Yang, 2000; Musienko et al., 2007; Hoogkamer et al., 2014; Cappellini et al., 2018). However, whether spinal locomotor networks without inputs from the brain can generate backward locomotion has received little to no attention. Human infants, with immature descending supraspinal pathways, can generate treadmill locomotion in forward and backward directions when supported (Lamb and Yang, 2000). In spinal animals, two studies reported that spinal kittens and adult cats could not perform backward locomotion on a treadmill (Robinson and Goldberger, 1986; Buford and Smith, 1990). However, two other studies showed hindlimb muscle activity and stance phases during backward locomotion on a treadmill in spinal animals that received pharmacological treatment and/or electrical epidural stimulation of the spinal cord (Barbeau et al., 1987; Courtine et al., 2009). The first study showed backward locomotion in a spinal cat treated with clonidine, an $\alpha-2$ noradrenergic agonist, and the other in a spinal rat treated with quipazine, a serotonergic agonist, that stepped in an upright position with epidural electrical spinal cord stimulation. Although these studies showed that spinal animals generated backward stepping movements on a treadmill, which was not their main goal, several questions remain unexplored.

In the present study, we ask four basic questions that address different fundamental aspects of the spinal control of backward locomotion. (1) Can spinal cats produce backward locomotion without increasing spinal cord excitability pharmacologically? We confirm and extend findings of Barbeau et al. (1987) in a larger sample of spinal cats that they can generate backward locomotion. However, we found backward locomotion required perineal stimulation, which increases spinal excitability through an undefined mechanism (Rossignol et al., 2006; Alluin et al., 2015; Harnie et al., 2019). (2) Can backward locomotion in spinal cats adjust to different treadmill speeds and to different speeds for the left and right hindlimbs on a split-belt treadmill? We test the hypothesis that sensory feedback from the limbs adjusts the output of the spinal CPG for backward locomotion to meet task demands. (3) Are spatiotemporal parameters, such as cycle and phase durations, stride and step lengths and paw placements, during backward locomotion similar in intact and spinal cats? Here, we test the hypothesis that sensorimotor circuits within the spinal cord adjust spatiotemporal parameters for backward locomotion. (4) Are spatiotemporal adjustments and patterns of muscle activity, including muscle synergies, for forward and backward locomotion in spinal cats similar or different? In intact cats and humans, some studies have reported similar muscle activation patterns and/or synergies for forward and backward locomotion, consistent with a shared locomotor CPG for both directions (Winter et al., 1989; Buford and Smith, 1990; Lamb and Yang, 2000), whereas others highlighted differences (Thorstensson, 1986; Grasso et al., 1998; Ivanenko et al., 2008), suggesting distinct functional networks. Here, we test the hypothesis that shared spinal sensorimotor circuits control muscle synergies for backward and forward locomotion.

\section{Materials and Methods}

\section{Animals and ethical information}

The Animal Care Committee of the Université de Sherbrooke approved all procedures (Protocol 442-18) in accordance with policies and directives of the Canadian Council on Animal Care. Six adult cats, three males and three females, weighing 3.6-4.7 kg were used in the present study. In our effort to reduce the number of animals used in research, we used these cats in other studies to answer different scientific questions (Harnie et al., 2019; Merlet et al., 2020). Our study followed the ARRIVE guidelines for animal studies (Kilkenny et al., 2010).

\section{Surgical procedures and electrode implantation}

We performed surgeries under aseptic conditions with sterilized instruments in an operating room. Before surgery, we injected butorphanol $(0.4 \mathrm{mg} / \mathrm{kg})$, acepromazine $(0.1 \mathrm{mg} / \mathrm{kg})$, and glycopyrrolate $(0.01 \mathrm{mg} / \mathrm{kg})$ intramuscularly for sedation and ketamine/diazepam $(0.05 \mathrm{ml} / \mathrm{kg})$ for induction. Cats were anesthetized with isoflurane (1.5-3\%) delivered in $\mathrm{O}_{2}$, first with a mask and then with an endotracheal tube. Cats received a continuous infusion of lactated Ringers solution ( $3 \mathrm{ml} / \mathrm{kg} / \mathrm{h}$ ) during surgery through a catheter placed in a cephalic vein. During surgery, we maintained anesthesia by adjusting isoflurane concentration as needed and by monitoring cardiac and respiratory rates. Body temperature was monitored with a rectal thermometer and maintained within physiological range $\left(37 \pm 0.5^{\circ} \mathrm{C}\right)$ using a water-filled heating pad placed under the animal and an infrared lamp $\sim 50 \mathrm{~cm}$ over it. We confirmed the depth of anesthesia by applying pressure to a paw (to detect limb withdrawal) and by assessing the size and reactivity of pupils. The animal's skin was shaved using electric clippers and cleaned with chlorhexidine soap. We directed pairs of Teflon-insulated multistrain fine wires (AS633; Cooner Wire) subcutaneously from two head-mounted 34-pin connectors (Omnetics Connector). Electrodes were sewn into the belly of selected hindlimb muscles for bipolar recordings, with 1-2 $\mathrm{mm}$ of insulation stripped from each wire. The head connector was secured to the skull using dental acrylic. We verified electrode placement during surgery by electrically stimulating each muscle through the appropriate head connector channel. At the end of surgery, we injected an antibiotic (Convenia, $0.1 \mathrm{ml} / \mathrm{kg}$ ) subcutaneously and taped a transdermal fentanyl patch $(25 \mathrm{mcg} / \mathrm{h})$ to the back of the animal $2-3 \mathrm{~cm}$ rostral to the base of the tail for prolonged analgesia (4- to 5-d period). We also injected buprenorphine $(0.01 \mathrm{mg} / \mathrm{kg})$, a fast-acting analgesic, subcutaneously at the end of the surgery and $\sim 7 \mathrm{~h}$ later. After surgery, we placed the cat in an incubator until they regained consciousness. At the conclusion of the experiments, cats received a lethal dose of pentobarbital through the cephalic vein, and we removed a section of the spinal cord around the lesion site for histologic analysis (Harnie et al., 2019).

\section{Spinal transection and hindlimb locomotor recovery}

For the spinal transection, surgical procedures were the same as in the previous section. The skin was incised over the last thoracic vertebrae and after carefully setting aside muscle and connective tissue, a small dorsal laminectomy was made. After exposing the spinal cord, we applied xylocaine (lidocaine hydrochloride, $2 \%$ ) topically and made two to three intraspinal injections. We then completely transected the spinal cord with surgical scissors between the 12th and 13th thoracic vertebrae. We then cleaned the $\sim 0.5-\mathrm{cm}$ gap between the two cut ends of the spinal cord and stopped any residual bleeding. We verified that no spinal cord tissue remained connecting rostral and caudal ends, which we later confirmed histologically. A hemostatic agent (Spongostan) was placed within the gap, and muscles and skin were sewn back to close the opening in anatomic layers. After spinal transection, we manually expressed the cat's bladder and large intestine one to two times daily, or as needed.

As stated previously, we used cats in another study to describe the effects of three interventions on the recovery of standing and hindlimb locomotion after spinal transection (Harnie et al., 2019). We use the same cat numbers as Harnie et al. (2019). In the present study, one cat (Cat 3) received manual therapy consisting of distal to proximal strokes of triceps surae muscles $(0.33 \mathrm{~Hz}, 10 \mathrm{~min}$ per leg, five times per week for five weeks). Four cats (Cats 5-8) received locomotor training (20 min, five times a week for five weeks) that consisted of two experimenters 

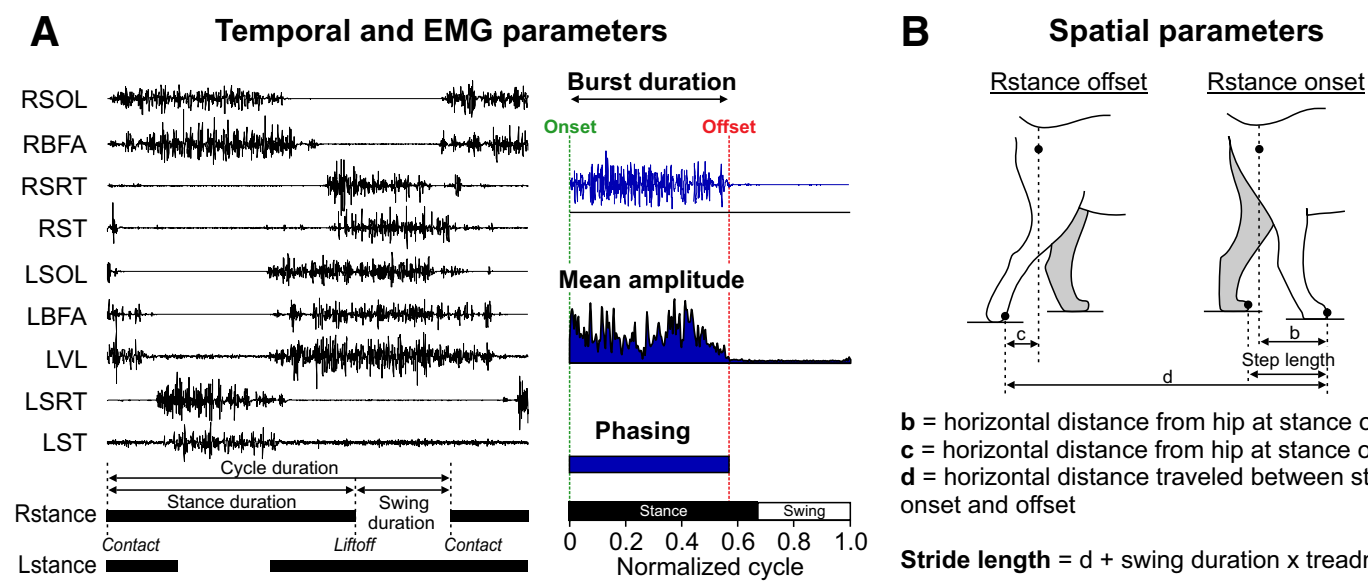

\begin{abstract}
$\mathbf{b}=$ horizontal distance from hip at stance onset $\mathbf{c}=$ horizontal distance from hip at stance offset $\mathbf{d}=$ horizontal distance traveled between stance onset and offset
\end{abstract}

Stride length $=d+$ swing duration $x$ treadmill speed

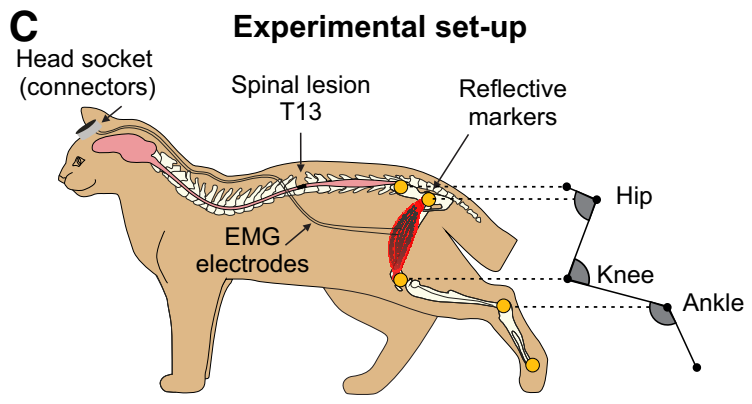

Figure 1. Experimental set-up and measured spatiotemporal parameters. $A$, Measured temporal and EMG variables. EMG activity is shown for nine muscles of the left (L) and right (R) hindlimbs during backward locomotion in an intact cat. Thick horizontal lines represent stance phases. SOL, soleus; BFA, biceps femoris anterior; VL, vastus lateralis; SRT, anterior sartorius; ST, semitendinosus. We measured EMG burst duration from onset to offset and mean EMG amplitude by integrating the full-wave rectified EMG burst from onset to offset divided by burst duration. We measured phasing as the onset and offset normalized to cycle duration. $\boldsymbol{B}$, Measured spatial parameters, including stride length, step length, and the horizontal distance of the paw at contact and liftoff relative to the hip. C, Experimental design showing EMG electrodes directed subcutaneously to muscles from a head connector and the site of the spinal transection. We placed reflective markers on bony landmarks to reconstruct measure joint angles.

moving the hindlimbs over the moving treadmill belt to reproduce locomotion, with one of the experimenters holding the tail for support. One cat (Cat 11) received no intervention. The main message of that study was that cats recovered hindlimb locomotion without or with treadmill training, indicating that the recovery of hindlimb locomotion simply requires a return of excitability within spinal sensorimotor circuits. Thus, cats in the present study are discussed in terms of their ability to generate hindlimb locomotion and not in terms of the intervention they received. It is important to note that none of the cats were trained to recover backward locomotion after spinal transection.

\section{Experimental design}

We collected data [electromyography (EMG) and kinematics] before (intact state) and six weeks after (spinal state) spinal transection during locomotion in the forward and backward directions on a split-belt treadmill, with the left and right sides on separate belts. In the intact state, cats performed tied-belt locomotion (equal left-right speeds) in quadrupedal and bipedal conditions. In the bipedal condition, the forelimbs were placed on a stationary platform. In the spinal state, cats performed tied-belt and split-belt (unequal left-right speeds) locomotion in the bipedal condition only. During tied-belt locomotion, both sides stepped from 0.1 to $0.5 \mathrm{~m} / \mathrm{s}$ in $0.1-\mathrm{m} / \mathrm{s}$ increments. During split-belt locomotion, the slow limb stepped at 0.1 or $0.2 \mathrm{~m} / \mathrm{s}$ and the fast limb stepped from 0.2 to $0.4 \mathrm{~m} / \mathrm{s}$ in $0.1-\mathrm{m} / \mathrm{s}$ increments. Both the left and right limbs were used as the slow and fast limbs during split-belt locomotion. Unless specified, we use the term "locomotion" for tied-belt locomotion. We collected data from five to 15 consecutive step cycles in each locomotor condition. In the spinal state, we collected data with and without perineal stimulation. For perineal stimulation, an experimenter manually pinched the skin under the tail with the index finger and thumb. We did not provide weight support, although an experimenter gently held the tail to provide equilibrium. To avoid fatigue, $20 \mathrm{~s}$ of rest were given between episodes of locomotion.
We collected kinematic data as described previously (Harnie et al., 2018, 2019). Videos of the left and right sides were obtained with two cameras (Basler AcA640-100 g) at 60 frames/s with a spatial resolution of $640 \times 480$ pixels. A custom-made program (Labview) acquired the images and synchronized acquisition with EMG data. By visual detection, we determined limb contact as the first frame where the paw made visible contact with the treadmill surface, and limb liftoff as the most caudal displacement of the toe, for both hindlimbs. We measured cycle duration from successive paw contacts, while stance duration corresponded to the interval of time from paw contact to liftoff (Fig. 1A). We measured swing duration as cycle duration minus stance duration.

EMG signals were preamplified $(10 \times$, custom-made system), bandpass filtered $(30-1000 \mathrm{~Hz})$, and amplified $(100-5000 \times)$ using a 16channel amplifier (model 3500; A-M Systems). As we implanted $>16$ muscles per cat, we obtained data in each locomotor condition twice, one for each connector, as our data acquisition system does not allow us to record $>16$ channels simultaneously. EMG data were digitized $(2000 \mathrm{~Hz})$ with a National Instruments card (NI 6032E), acquired with custom-made acquisition software and stored on computer. We analyzed data from the following muscles listed alphabetically: biceps femoris anterior (BFA; hip extensor), biceps femoris posterior (BFP; knee flexor/hip extensor), caudofemoralis (CF; hip extensor), tensor fascia latae (FL; hip flexor/hip abductor), iliopsoas (IP; hip flexor), gluteus maximus (GLU; hip extensor), lateral gastrocnemius (LG; ankle extensor/knee flexor), medial gastrocnemius (MG; ankle extensor/knee flexor), plantaris (PLA; ankle extensor/knee flexor), peroneus longus (PLO; ankle extensor/foot eversion), sartorius anterior (SRT; hip flexor/knee extensor), semimembranosus (SM; hip extensor), semitendinosus (ST; knee flexor/hip extensor), soleus (SOL; ankle extensor), tibialis anterior (TA; ankle flexor), and vastus lateralis (VL; knee extensor). The same experimenter (J.H.) determined burst onsets and offsets by visual inspection from the raw EMG waveforms using a custommade program. Burst duration was measured from onset to offset. 
Mean EMG amplitude was measured by integrating the full-wave rectified EMG burst from onset to offset and dividing it by its burst duration (Fig. 1A).

Stride length was measured as the horizontal distance traveled from liftoff to contact plus the distance traveled by the treadmill belt during the swing phase (swing duration multiplied by treadmill speed; Courtine et al., 2005; Goetz et al., 2012; Thibaudier and Frigon, 2014; Harnie et al., 2018; Fig. 1B). To reconstruct joint kinematics for the hindlimbs, we placed reflective markers over the iliac crest, greater trochanter, lateral malleolus, metatarsophalangeal (MTP) joint and at the tip of the toes. Frame-by-frame reconstruction of the videos allowed us to visualize the overall movement of the hindlimbs in a stick figure format by connecting each joint sequentially (Fig. 1C).

Characterization of muscle synergies

We determined muscle synergies and their time-dependent activation patterns for forward and backward locomotion at $0.3 \mathrm{~m} / \mathrm{s}$ in spinal cats using the non-negative matrix factorization (NNMF) algorithm (Cheung et al., 2005; Ting and McKay, 2007; Tresch and Jarc, 2009). We used EMG signals from multiple muscles recorded in three cats (Cat 11, 10 muscles; Cat 5, 15 muscles; and Cat 3, 13 muscles). The algorithm (MATLAB function $n n m f$ ) decomposes experimental EMG envelopes, arranged in a $m \times T$ matrix $\mathbf{D}$, into a $m \times n$ matrix of muscle synergy weights $\mathbf{W}$ (columns in $\mathbf{W}$ that specify relative muscle contribution to each synergy) and a $n \times T$ matrix $\mathbf{C}$ of time-dependent activation coefficients for each synergy; that is, $\mathbf{D}=\mathbf{W C}+$ error. Here, $T=100$, the number of time samples in each EMG envelope in a cycle; $m$ is the number of recorded muscles in each cat and locomotor direction; $n$ is the number of synergies. To obtain matrix $\mathbf{D}$ for each cycle, we rectified the bandpass-filtered EMG signals, determined for each muscle the EMG burst threshold separating the EMG baseline (interburst intervals) from EMG bursts (typically using 2 SDs from the mean baseline magnitude), and substituted the baseline signal with zero values. We filtered the obtained signals using a low-pass, zero-lag Butterworth filter at $6 \mathrm{~Hz}$ and divided the continuous EMG envelopes into separate cycles based on video recordings. We normalized the duration of each cycle to $100 \%$ and the magnitude of each muscle low-pass filtered EMG to the maximum value selected across all cycles of forward and backward locomotion for a given muscle and cat. We performed muscle synergy analysis for each of the three cats and each locomotion cycle in which EMG activity was recorded synchronously in all available muscles. Because the NNMF is a stochastic algorithm (Lee and Seung, 1999), we applied the algorithm 20 times for each cycle. As the number of muscle synergies that accurately reproduces experimental EMG patterns (matrix D) from the extracted matrices $\mathbf{W}$ and $\mathbf{C}$ was a priori not known, we performed extractions of matrices $\mathbf{W}$ and $\mathbf{C}$ for each obtained matrix $\mathbf{D}$ assuming a different number of synergies from 1 to 10 . We then computed for each extraction the reconstructed EMG patterns (matrix $\mathbf{D}^{*}=\mathbf{W C}$ ) and the amount of variance accounted for by $\mathbf{D}^{*}$ in $\mathbf{D}\left(R^{2}\right)$. Because the NNMF does not provide a unique order of synergies (order of columns in $\mathbf{W}$, or rows in $\mathbf{C}$ ) in each decomposition of matrix $\mathbf{D}$, we sorted the computed synergies in all extractions based on the maximum correlation $\left(R^{2}\right)$ of their timevarying activation coefficients.

To test common muscle synergies for forward and backward locomotion, we needed to extract these synergies from a matrix $\mathbf{D}_{\mathrm{COM}}$ that contained the recorded EMG patterns of both forward and backward locomotion, and to compare the obtained "common" synergies with direction-specific synergies extracted from matrices $\mathbf{D}_{\mathrm{FW}}$ and $\mathbf{D}_{\mathrm{BW}}$. We also wanted to compare extracted muscle synergies among animals. We could not perform these analyses in individual cats because the sets and numbers of recorded muscles were different in some cats and locomotor directions. These differences could potentially affect composition and patterns of computed synergies. For example, relatively fewer recorded extensor muscles would likely reduce the number of obtained extensor synergies. Therefore, we performed an additional synergy analysis. For this analysis, we selected EMG patterns from the same muscles $(m=7)$ recorded in all three cats during forward and backward locomotion. We extracted muscle synergies spanning both forward and backward locomotion (a $m \times n$ matrix $\mathbf{W}_{\mathrm{COM}}$ of muscle weights and a $n \times 2 T$ matrix
Table 1. Comparison of recorded and reconstructed muscle activation patterns during forward and backward locomotion

\begin{tabular}{llll}
\hline Recorded EMG & Reconstructed EMG & $R^{2}$ & RMSD \\
\hline $\mathbf{D}_{\mathrm{FW}}$ & $\mathbf{D}_{1}^{*}=\mathbf{W}_{\mathrm{FW}} \mathbf{C}_{\mathrm{FW}}$ & 0.947 & 0.051 \\
$\mathbf{D}_{\mathrm{FW}}$ & $\mathbf{D}_{2}^{*}=\mathbf{W}_{\mathrm{BW}} \mathbf{C}_{\mathrm{FW}}$ & $0.687 *$ & $0.108 *$ \\
$\mathbf{D}_{\mathrm{FW}}$ & $\mathbf{D}_{3}^{*}=\mathbf{W}_{\mathrm{FW}} \mathbf{C}_{\mathrm{BW}}$ & $0.800 *$ & $0.119 *$ \\
$\mathbf{D}_{\mathrm{FW}}$ & $\mathbf{D}_{4}^{*}=\mathbf{W}_{\mathrm{COM}} \mathbf{C}_{\mathrm{COMFW}}$ & 0.858 & $0.075 *$ \\
$\mathbf{D}_{\mathrm{FW}}$ & $\mathbf{D}_{5}^{*}=\mathbf{W}_{\mathrm{COM}} \mathbf{C}_{\mathrm{FW}}$ & $0.821 *$ & $0.087 *$ \\
$\mathbf{D}_{\mathrm{FW}}$ & $\mathbf{D}_{6}^{*}=\mathbf{W}_{\mathrm{FW}} \mathbf{C}_{\mathrm{COMFW}}$ & 0.946 & $0.797 *$ \\
$\mathbf{D}_{\mathrm{FW}}$ & $\mathbf{D}_{7}^{*}=\mathbf{W}_{\mathrm{BW}} \mathbf{C}_{\mathrm{BW}}$ & 0.967 & 0.056 \\
$\mathbf{D}_{\mathrm{FW}}$ & $\mathbf{D}_{8}^{*}=\mathbf{W}_{\mathrm{FW}} \mathbf{C}_{\mathrm{BW}}$ & $0.593 * *$ & $0.149 * *$ \\
$\mathbf{D}_{\mathrm{FW}}$ & $\mathbf{D}_{9}^{*}=\mathbf{W}_{\mathrm{BW}} \mathbf{C}_{\mathrm{FW}}$ & $0.737 * *$ & $0.151 * *$ \\
$\mathbf{D}_{\mathrm{FW}}$ & $\mathbf{D}_{1}^{*}=\mathbf{W}_{\mathrm{COM}} \mathbf{C}_{\mathrm{COMBW}}$ & 0.870 & 0.087 \\
$\mathbf{D}_{\mathrm{FW}}$ & $\mathbf{D}_{11}^{*}=\mathbf{W}_{\mathrm{COM}} \mathbf{C}_{\mathrm{BW}}$ & $0.752 * *$ & $0.121 * *$ \\
$\mathbf{D}_{\mathrm{FW}}$ & $\mathbf{D}_{12}^{*}=\mathbf{W}_{\mathrm{BW}} \mathbf{C}_{\mathrm{COMBW}}$ & $0.797 * *$ & $0.111 * *$ \\
\hline
\end{tabular}

The table shows values comparing EMG patterns recorded during forward (matrix $\mathbf{D}_{\mathrm{FW}}$ ) and backward (matrix $D_{\mathrm{FW}}$ ) locomotion with EMG patterns reconstructed from the corresponding five synergies using different combinations of muscle synergy weights (matrices $\mathbf{W}_{\mathrm{FW}}, \mathbf{W}_{\mathrm{BW}}$, and $\mathbf{W}_{\mathrm{COM}}$ ) and time-varying activation coefficients $\left(\mathbf{C}_{\mathrm{FW}}, \mathbf{C}_{\mathrm{BW}}, \mathbf{C}_{\mathrm{COM} \_\mathrm{FW}}\right.$, and $\left.\mathbf{C}_{\mathrm{COM} \_\mathrm{FW}}\right)$. Note: $R^{2}$ is the variance accounted for in recorded EMG patterns by reconstructed EMG patterns. RMSD is the root mean square difference calculated between the recorded and reconstructed patterns across all muscles and normalized time samples. $*$, statistically different $(p \leq 0.01)$ from condition $\mathbf{D}_{1}^{*}=\mathbf{W}_{\mathrm{FW}} \mathbf{C}_{\mathrm{FW}} ; * *$, statistically different $(p \leq 0.01)$ from condition $\mathbf{D}_{7}^{*}=\mathbf{W}_{\mathrm{BW}} \mathbf{C}_{\mathrm{BW}}$.

$\mathrm{C}_{\mathrm{COM}}$ of time-dependent activation coefficients) from a $m \times 2 \mathrm{~T}$ matrix $\mathbf{D}_{\mathrm{COM}}$, containing EMG patterns of forward and backward locomotion; that is, $\mathbf{D}_{\mathrm{COM}}=\mathbf{W}_{\mathrm{COM}} \mathbf{C}_{\mathrm{COM}}+$ error (where $m$ is the number of muscles, $n$ is the number of synergies, and $T$ is the number of time samples in each EMG pattern in a cycle, $T=100$ ). Matrices $\mathbf{C}_{\mathrm{COM}}$ and $\mathbf{D}_{\mathrm{COM}}$ had $2 T$ columns; the first $T$ columns corresponded to 100 time samples of EMG patterns of forward locomotion and the next $T$ columns corresponded to samples of backward locomotion for each muscle $m$. Thus, matrix $\mathbf{C}_{\mathrm{COM}}$ consisted of two $n \times T$ matrices $\mathbf{C}_{\mathrm{COM} \_ \text {Fw }}$ and $\mathbf{C}_{\mathrm{COM} \_ \text {BW. }}$. To determine whether matrices spanning both forward and backward locomotion ( $\mathbf{W}_{\mathrm{COM}}$ and $\mathbf{C}_{\mathrm{COM}}$ ) accurately reconstructed matrices containing EMG patterns for forward $\left(\mathbf{D}_{\mathrm{FW}}\right)$ and backward $\left(\mathbf{D}_{\mathrm{BW}}\right)$ locomotion, we reconstructed the EMG patterns from different combinations of extracted synergies and their time-varying activation coefficients (Table 1).

\section{Statistical analysis}

We performed statistical analyses using IBM SPSS Statistics 24.0 software. Results presented in Figures 3-5 were analyzed using mixed linear models (MLM) for repeated measures. With this approach, we identified significant differences by considering condition as the sole factor (Fig. 3) or both condition and speed as factors (Figs. 4, 5). We verified the assumption of approximately normally distributed residuals and assessed statistical significance of predictors at a significant level of 0.01 . For the data presented in Figure 6, we performed a paired $t$ test with a significance level of 0.01 .

We tested significance of the difference in muscle synergy weights (the columns in matrix $\mathbf{W}$ ) between forward and backward locomotion in each of three spinal cats (Fig. 7). For that purpose, we used the MLM analysis with two independent factors: locomotor direction (forward and backward) and muscle. We conducted a similar MLM analysis for muscle synergies obtained for the three cats together and for synergies spanning both forward and backward locomotion (Fig. 8). In this analysis, we used three independent factors: locomotor direction (forward, backward and combined), muscle and cat. To test the similarity of the timevarying activation coefficients for each synergy (rows in matrix C) between the forward, backward and combined conditions, we averaged the coefficients for every $10 \%$ time bin of the cycle and applied the MLM analysis with the independent factors of locomotor direction, binned time or cat. In addition, we computed the coefficient of determination $\left(R^{2}\right)$ between the pairs of the mean time-varying activation patterns corresponding to the forward $\left(\mathrm{C}_{\mathrm{FW}}\right)$, backward $\left(\mathrm{C}_{\mathrm{BW}}\right)$, and combined directions $\left(\mathbf{C}_{\mathrm{COM} \_ \text {FW }}\right.$ and $\left.\mathbf{C}_{\mathrm{COM} \_ \text {BW }}\right)$ for each synergy (Table 1; Fig. 8). We set significance level at 0.01 . 
FW-Biped-Spinal
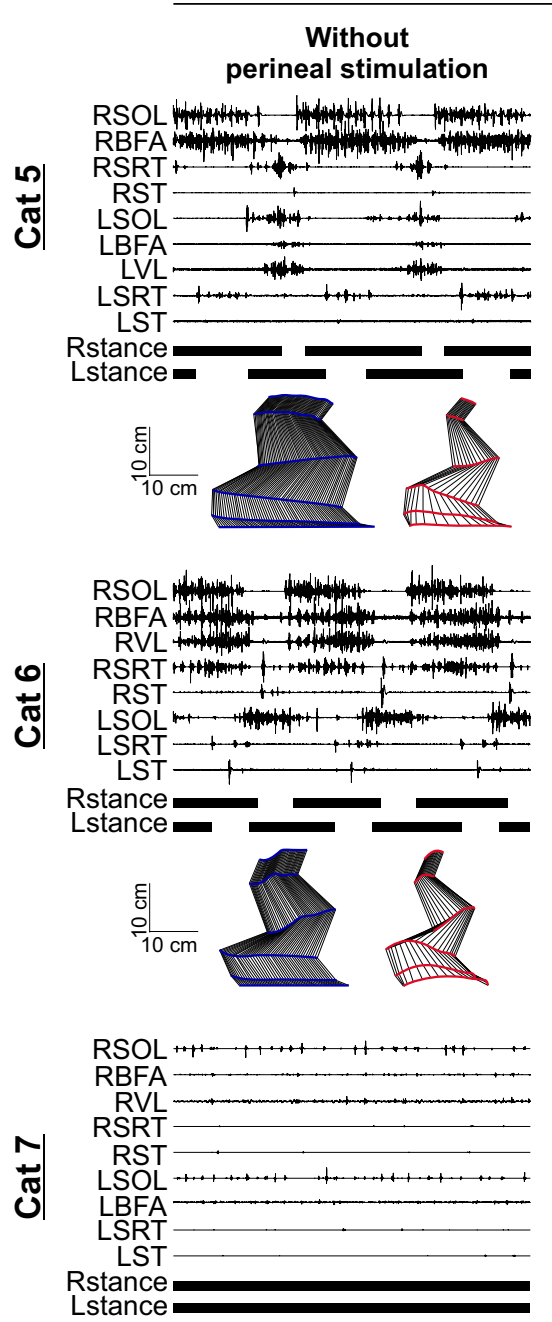

동|
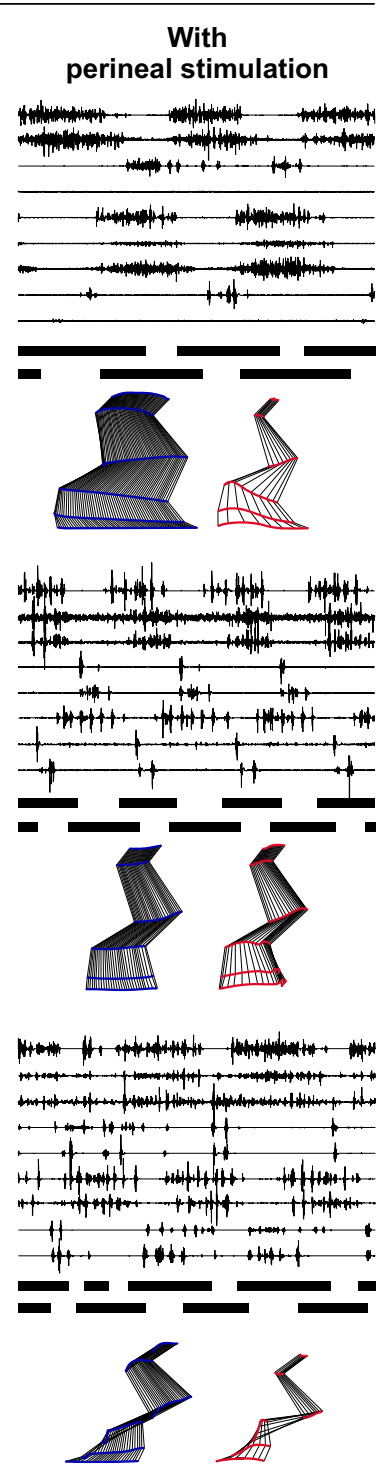

BW-Biped-Spinal
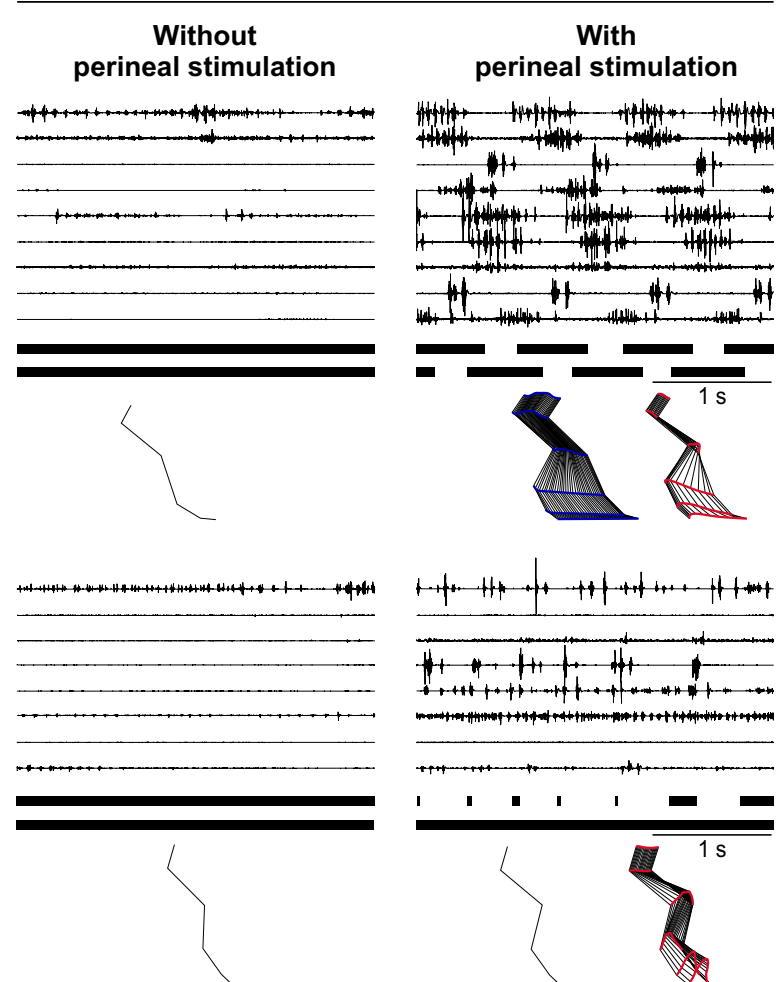

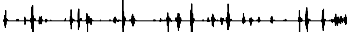

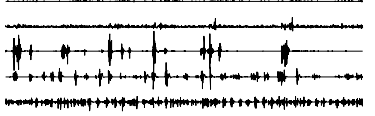

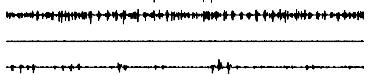
$\frac{1 \text { I I I }}{-1 \mathrm{~s}}$ $\overbrace{\infty}^{1 s}$
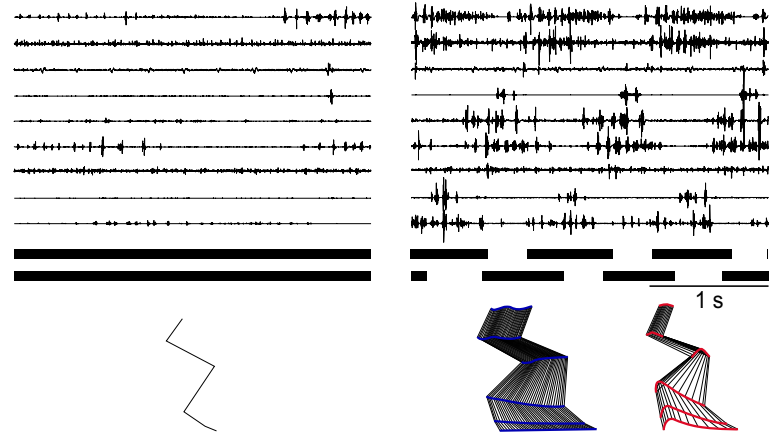

Figure 2. Presence and absence of forward and backward locomotion in three spinal cats. The top, middle and bottom panels show EMG activity from eight to nine hindlimb muscles along with stance phases (horizontal thick lines) of the left (L) and right (R) hindlimbs in three spinal cats during forward (FW-biped-spinal) and backward (BW-biped-spinal) bipedal locomotion without and with perineal stimulation. For muscle abbreviations, see Figure 1. Stick figures show hindlimb kinematics of the right hindlimb for a representative cycle.

\section{Results}

All cats recovered forward and/or backward locomotion following spinal transection, as described below. We confirmed histologically that the spinal transection was complete in all six cats, as shown in Harnie et al. (2019; their Fig. 1C), where cats of the present study have the same number.

Backward locomotion after spinal transection requires an increase in spinal neuronal excitability

Several studies have characterized backward locomotion in the intact cat, including at different speeds (Buford and Smith, 1990, 1993; Buford et al., 1990; Perell et al., 1993; Pratt et al., 1996). The first question we asked was whether spinal cats, without descending commands from the brain, can perform backward locomotion on a treadmill. As stated in the introduction, Barbeau et al. (1987) mentioned that spinal cats could perform backward locomotion and showed alternating activity in two hindlimb muscles bilaterally along with stance and swing phases in a single cat at a treadmill speed of $0.1 \mathrm{~m} / \mathrm{s}$ (Barbeau et al. 1987, their Fig. 7). They also anecdotally reported that clonidine, an $\alpha-2$ noradrenergic agonist, facilitated backward locomotion in spinal cats. Here, we confirm, in a larger sample, that spinal cats can perform backward locomotion up to speeds of $0.5 \mathrm{~m} / \mathrm{s}$ but only with strong perineal stimulation provided by an experimenter. Tonically applied perineal stimulation increases spinal neuronal excitability and facilitates hindlimb locomotion in spinal mammals through an undefined mechanism (Bélanger et al., 1996; Leblond et al., 2003; Alluin et al., 2015; Harnie et al., 2019). Without perineal stimulation, the hindlimbs simply dragged forward rostral to the hip. In addition, perineal stimulation had to be started while spinal cats were standing, in other words, before the treadmill belt started moving. If the hindlimbs remained stuck in a rostral position, we had to stop the treadmill belt, replace the animal in a standing position and restart the trial. Interestingly, some spinal cats could only step in one direction, either forward or backward. Figure 2 shows the EMG activity of selected hindlimb muscles along with the stance phases during 
A
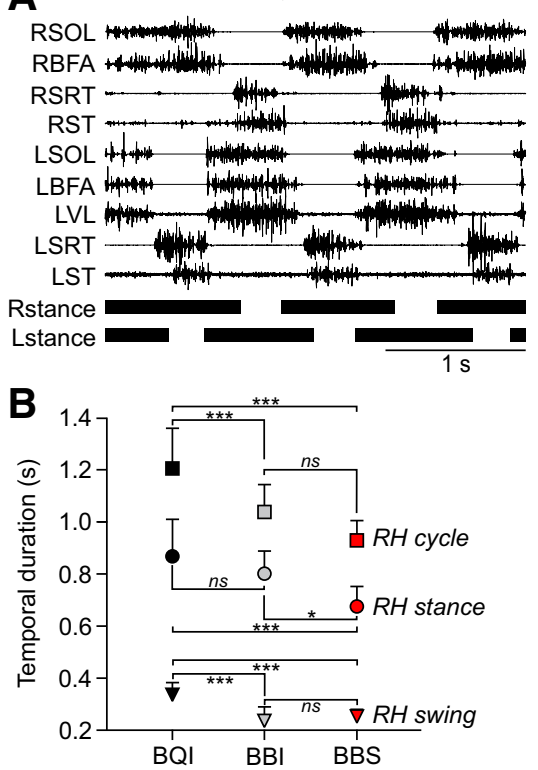

E

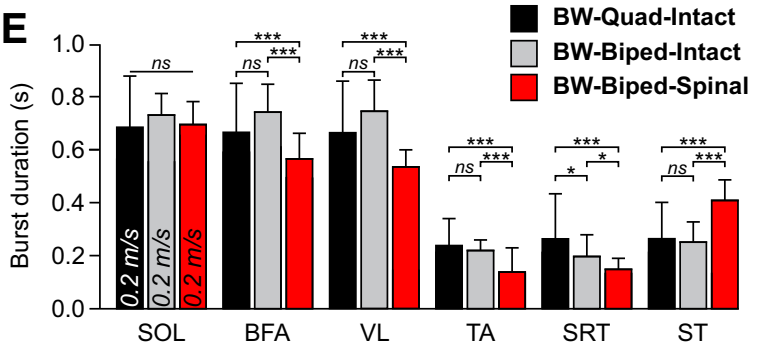

BW-Biped-Intact

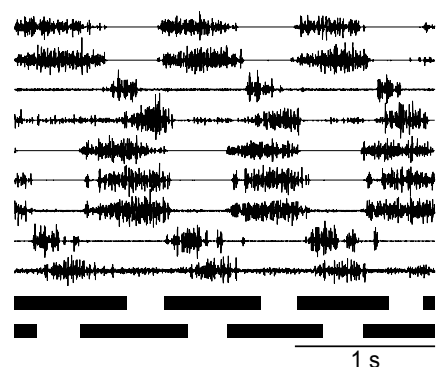

$\mathbf{C}_{30}$
BW-Biped-Spinal

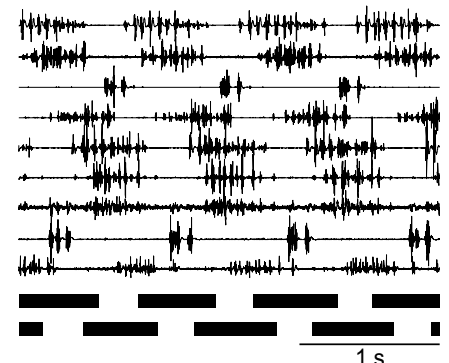

D

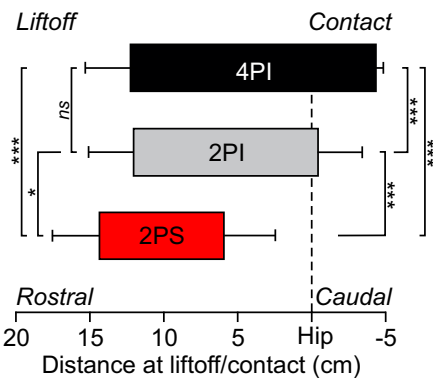

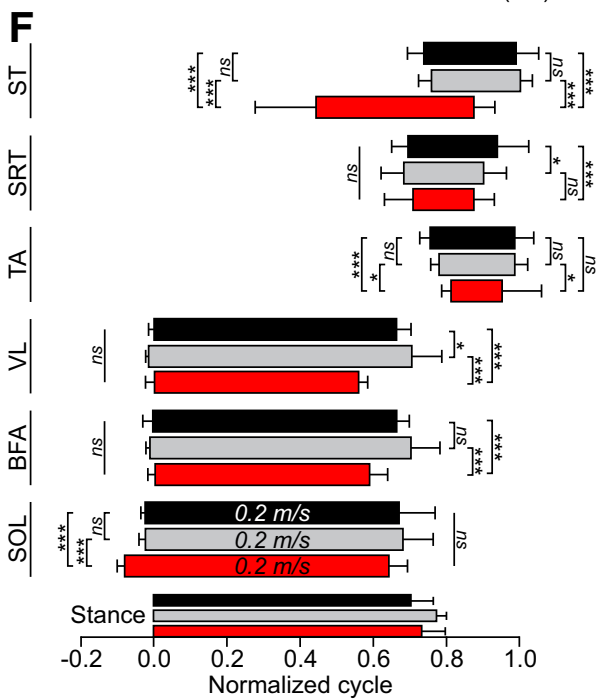

Figure 3. Backward quadrupedal or bipedal locomotion in intact and spinal cats at $0.2 \mathrm{~m} / \mathrm{s}$. $\boldsymbol{A}$, EMG activity from nine hindlimb muscles along with stance phases (horizontal thick lines) of the left (L) and right (R) hindlimbs in one cat in the intact state during quadrupedal (BW-quadrupedal-intact or BQI) and bipedal (BW-biped-intact or BBI) backward locomotion and after spinal transection in the bipedal condition (BW-biped-spinal or BBS). Data are from (at 5 before and six weeks after spinal transection. For muscle abbreviations, see Figure 1. B, Cycle, stance and swing durations in the three conditions. $\boldsymbol{C}$, Stride and step lengths in the three conditions. $\boldsymbol{D}$, Distance at contact and liftoff in the three conditions. EMG burst durations $(\boldsymbol{E})$ and phasing $(\boldsymbol{F})$ in the three conditions. $\boldsymbol{B}-\boldsymbol{F}$, Each data point or bar is the mean \pm SD for the group ( $n=3$ cats), whereas statistical analyses were done on pooled cycles/bursts. BQI ( $n=20$ cycles/bursts), BBI ( $n=29$ cycles/bursts), BBS ( $n=34$ cycles/bursts). Asterisks indicate a significant effect of condition (MLM) at $* p<0.01, * * p<0.001, * * * p<0.0001 ;$ ns, not significant.

forward and backward locomotion in three spinal cats at $0.2 \mathrm{~m} / \mathrm{s}$ without and with perineal stimulation. Cat 5 stepped equally well during forward locomotion without and with perineal stimulation (Fig. 2, top panel). However, backward locomotion required perineal stimulation. Cat 6 had a robust hindlimb pattern during forward locomotion without perineal stimulation that was slightly impaired by light perineal stimulation (Fig. $2 B$, middle panel). Moderate to strong perineal stimulation disrupted the forward locomotor pattern in Cat 6 (data not shown). This cat could not perform backward locomotion with or without perineal stimulation. In contrast, Cat 7 could not perform forward locomotion without or with perineal stimulation but performed backward locomotion with perineal stimulation (Fig. $2 C$, bottom panel). When present (five of six spinal cats), backward locomotion was only expressed with perineal stimulation.
Backward locomotion in spinal cats more closely resembles backward bipedal locomotion in intact cats

When evaluating bipedal forward locomotion in spinal cats, studies have mostly compared the hindlimb pattern with the one obtained during quadrupedal locomotion in intact cats (Bélanger et al., 1996; Frigon and Rossignol, 2008; Desrochers et al., 2019; see however de Guzman et al., 1991; de Leon et al., 1998). Here, we compared backward bipedal locomotion in spinal cats (BW-biped-spinal) with the hindlimb pattern obtained during quadrupedal (BW-quad-intact) and bipedal (BW-biped-intact) backward locomotion in the intact state (Fig. 3). Figure $3 A$ shows the EMG activity of nine selected hindlimb muscles along with the right and left hindlimb stance phases during backward quadrupedal and bipedal locomotion in the intact state and in the same cat during backward bipedal locomotion in the spinal state. 
The EMG activity was similar in both conditions in the intact state, whereas it was more clonic in the spinal state, although burst timing was similar overall.

Cycle $\left(p=5.17^{\mathrm{e}-8}\right)$, stance $\left(p=9.96^{\mathrm{e}-6}\right)$, and swing $\left(p=1.04^{\mathrm{e}-7}\right)$ durations were significantly different between the three conditions $(p<0.01$ MLM; Fig. 3B). For cycle, stance and swing, we observed the longest durations in the BW-quad-intact condition followed in general by BW-biped-intact and BW-biped-spinal. Stride length $\left(p=1.12^{\mathrm{e}-18}\right)$ but not step length $(p=0.408)$ was significantly different between conditions, indicating the distance traveled during a stride differed but not the spacing between the hindlimbs at contact of the leading limb ( $p<0.01$ MLM; Fig. 3C). We observed the longest stride lengths in the BW-quad-intact condition followed by BW-biped-intact and BW-biped-spinal conditions. Distance at contact $\left(p=1.53^{\mathrm{e}-27}\right)$ and liftoff $\left(p=5.89^{\mathrm{e}-4}\right)$ were significantly different between the three conditions $(p<0.01$ MLM; Fig. $3 D$ ). The hindpaw was significantly more rostral to the hip at liftoff and contact in the BW-biped-spinal condition compared with the two conditions in the intact state. Indeed, the hindpaw did not travel caudal to the hip at contact in the spinal state.

The EMG burst durations significantly differed between conditions for BFA $\left(p=7.35^{\mathrm{e}-9}\right)$, VL $\left(p=3.89^{\mathrm{e}-13}\right)$, TA $\left(p=5.02^{\mathrm{e}-13}\right)$, SRT $\left(p=9.07^{\mathrm{e}-8}\right)$, and ST $\left(p=2.63^{\mathrm{e}-17}\right)$ muscles but not for SOL $(p=0.332$; Fig. $3 E)$. We observed a significantly shorter burst duration in the BW-biped-spinal condition for BFA, VL, and SRT compared with the two conditions in the intact state. On the other hand, ST burst duration was significantly prolonged in the BW-biped-spinal condition. When looking at EMG burst phasing, we found a significantly earlier onset of SOL in the BWbiped-spinal condition, whereas the hip extensor BFA and knee extensor VL did not have an earlier onset, although they terminated significantly earlier in the BW-biped-spinal condition (Fig. $3 F$ ). The most striking change occurred in the ST muscle in the BW-biped-spinal condition, which started bursting from mid- to late stance into mid-swing whereas in the conditions in the intact state, it burst from early to late swing. This earlier onset could be because of the greater stretch of the ST as the paw traveled more rostrally in the BW-biped-spinal condition or possibly to assist in weight support. The onset of the SRT burst did not differ between conditions, starting in late stance/early swing, although it terminated earlier in the BW-biped-spinal condition. We observed later onset and earlier offset of TA in the BW-bipedspinal condition.

\section{Backward locomotion in spinal cats adjusts to an increase in treadmill speed}

In the previous section, we showed that spatiotemporal measures in spinal cats during bipedal backward locomotion were closer in value to those observed during bipedal backward locomotion in intact cats. In this section, we compare adjustments to an increase in speed during bipedal backward locomotion in intact (BW-biped-intact) and spinal (BW-biped-spinal) cats (Fig. 4). Figure $4 A$ shows the EMG activity of 10 selected hindlimb muscles along with right and left hindlimb stance phases during backward bipedal locomotion at $0.1,0.2$, and $0.3 \mathrm{~m} / \mathrm{s}$ in the same cat (Cat 5) in the intact and spinal states. The EMG burst durations of extensor muscles decreased with increasing speed along with the stance phases in both states, with more clonic EMG observed in the spinal state.

We found that cycle $\left(p=3.19^{\mathrm{e}-51}\right)$ and stance $\left(p=1.80^{\mathrm{e}-52}\right)$ durations during backward bipedal locomotion were significantly reduced by increasing treadmill speed in both states while swing was unaffected ( $p=0.429$; Fig. $4 B$ ). On average, cycle duration was slightly but significantly longer in the intact state compared with the spinal state $(p=0.002 ; 0.1$ s or $9.2 \%$ difference). On average, stance duration was slightly but significantly longer in the intact state compared with the spinal state ( $p=1.01^{\mathrm{e}-3} ; 0.14 \mathrm{~s}$ or $14.1 \%$ difference). We also found that swing duration was slightly longer in the spinal state on average ( $p=0.002$; 0.02 s or $11.8 \%$ difference).

In intact and spinal cats, stride $\left(p=6.86^{\mathrm{e}-38}\right)$ and step $\left(p=2.70^{\mathrm{e}-13}\right)$ lengths significantly increased with increasing speed during backward bipedal locomotion (Fig. $4 C$ ). We found that stride length was significantly longer in the intact state on average $\left(p=7.42^{\mathrm{e}-12} ; 2.46 \mathrm{~cm}\right.$ or $14.0 \%$ difference). On the other hand, we found that step length was significantly longer in the spinal state on average $\left(p=3.31^{\mathrm{e}-4} ; 1.90 \mathrm{~cm}\right.$ or $26.9 \%$ difference). In intact and spinal cats, the distance of the hindpaw relative to the hip at liftoff was significantly more rostral to the hip with increasing speed $\left(p=3.69^{\mathrm{e}-7} ;\right.$ Fig. $\left.4 D\right)$. In contrast, the distance at contact relative to the hip was unaffected by speed $(p=0.247)$. When comparing the two states, spinal cats executed their liftoff ( $p=1.44^{\mathrm{e}-9} ; 1.86 \mathrm{~cm}$ or $15.3 \%$ difference) and made contact $\left(p=1.62^{\mathrm{e}-45} ; 6.33 \mathrm{~cm}\right.$ or $2503.3 \%$ difference) significantly more rostral to the hip compared with intact cats.

In intact and spinal cats, EMG burst durations of extensor muscles, namely SOL $\left(p=2.23^{\mathrm{e}-45}\right)$, BFA $\left(p=4.61^{\mathrm{e}-65}\right)$, and VL $\left(p=5.08^{\mathrm{e}-55}\right)$ significantly decreased with increasing speed during backward bipedal locomotion (Fig. $4 E$ ). On average, extensor EMG burst duration was significantly longer in intact cats compared with spinal cats for BFA $\left(p=9.74^{\mathrm{e}-20} ; 0.2\right.$ s or $23.4 \%$ difference) and VL $\left(p=2.48^{\mathrm{e}-13} ; 0.23 \mathrm{~s}\right.$ or $21.9 \%$ difference) but not SOL $(p=0.857)$. The EMG burst duration of TA and SRT did not change with speed ( $p=0.195$ and $p=0.714$, respectively) but was slightly longer in intact cats $\left(p=1.34^{\mathrm{e}-17} ; 0.07 \mathrm{~s}\right.$ or $32.9 \%$ difference; $p=1.22^{\mathrm{e}-10} ; 0.05 \mathrm{~s}$ or $26.4 \%$ difference, respectively) compared with spinal cats. We found that the EMG burst duration of the knee flexor/hip extensor ST muscle changed with speed $(p=0.01)$, albeit not consistently, and was slightly longer in spinal cats $\left(p=1.36^{\mathrm{e}-9} ; 0.1 \mathrm{~s}\right.$ or $37.6 \%$ difference).

When looking at the phasing of EMG bursts, we found a significant shift in onsets and offsets with increasing speed for all muscles in intact and spinal cats during backward bipedal locomotion (Fig. 4F). Within a normalized cycle, onsets occurred significantly earlier at faster speeds for SOL $\left(p=1.93^{\mathrm{e}-4}\right)$, BFA $(p=0.001)$, VL $\left(p=9.41^{\mathrm{e}-4}\right)$, TA $\left(p=5.47^{\mathrm{e}-17}\right)$, SRT $\left(p=3.51^{\mathrm{e}-28}\right)$, and ST $\left(p=9.75^{\mathrm{e}-18}\right)$. On average, we observed an earlier onset in spinal cats compared with intact cats for SOL $\left(p=3.47^{\mathrm{e}-17} ; 6.9 \%\right.$ difference) and ST ( $p=2.61^{\mathrm{e}-20} ; 21.7 \%$ difference) while a later onset was found for BFA $\left(p=3.93^{\mathrm{e}-15} ; 5.3 \%\right.$ difference $)$, VL $\left(p=1.66^{\mathrm{e}-7} ; 2.4 \%\right.$ difference), and SRT $(p=0.002 ; 2.3 \%$ difference). Onset of TA did not significantly differ between conditions ( $p=0.109)$. Offsets occurred significantly earlier at faster speeds for SOL $\left(p=4.02^{\mathrm{e}-25}\right)$, BFA $\left(p=4.59^{\mathrm{e}-29}\right)$, VL $\left(p=1.15^{\mathrm{e}-23}\right)$, SRT $\left(p=1.79^{\mathrm{e}-5}\right)$, and ST $(p=0.004)$. On average, we observed an earlier offset in spinal cats compared with intact cats for BFA $\left(p=2.94^{\mathrm{e}-16} ; 8.8 \%\right.$ difference $)$, VL $\left(p=8.01^{\mathrm{e}-10} ; 8.6 \%\right.$ difference $)$, TA $\left(p=4.81^{\mathrm{e}-10} ; 5.5 \%\right.$ difference $)$, and ST $\left(p=7.74^{\mathrm{e}-8} ; 10.4 \%\right.$ difference) but not for SOL $(p=0.195)$ and SRT $(p=0.097)$.

\section{Forward and backward locomotion in spinal cats show similar spatiotemporal adjustments to an increase in treadmill speed}

In the previous section, we observed that intact and spinal cats performed similar spatiotemporal and EMG adjustments to an increase in treadmill speed during bipedal backward locomotion, 
A
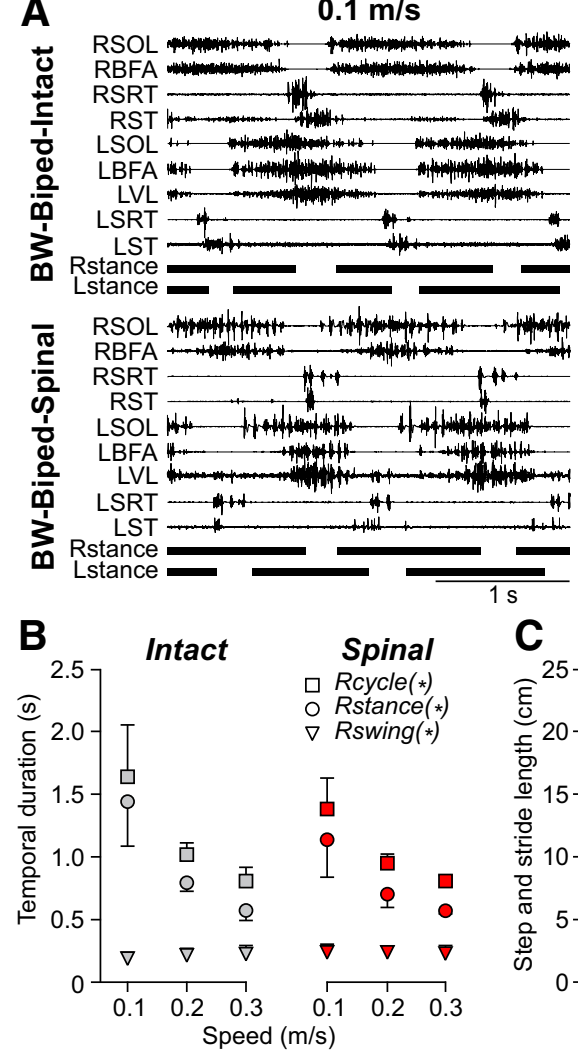

E

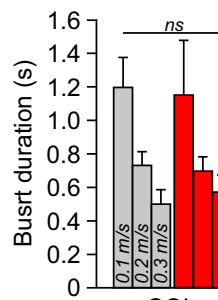

$\mathrm{SOL}$

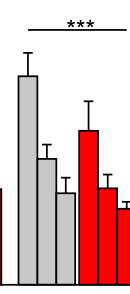

BFA

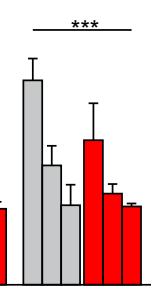

VL

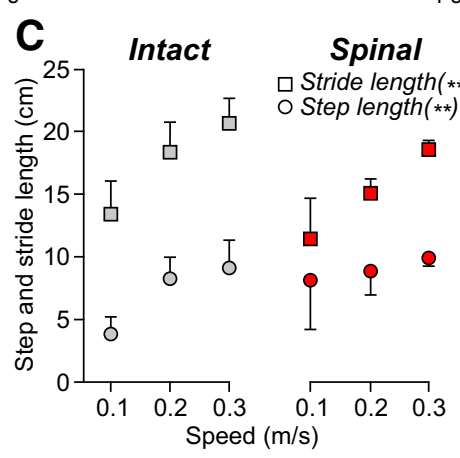

$\mathrm{C}$
$0.2 \mathrm{~m} / \mathrm{s}$

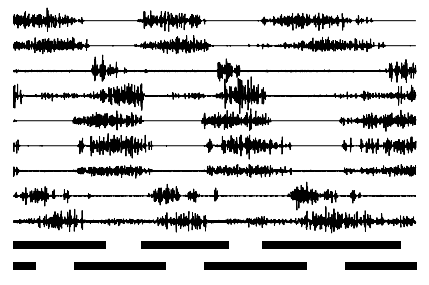

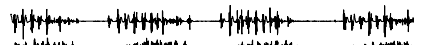
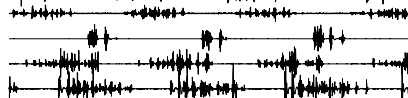

(t)

(.)

nt-

$=\square$

D

Liftoff(***) Contact(***)

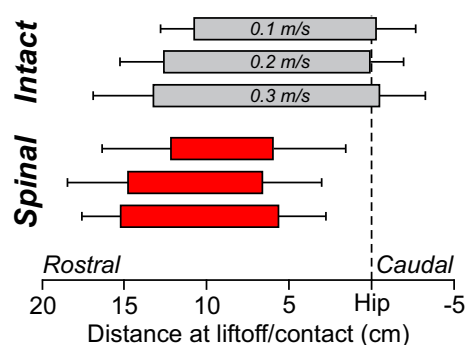

$\mathbf{F}$

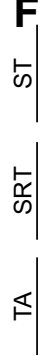

BW-Biped-Intact

BW-Biped-Spinal

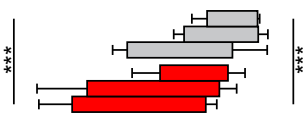

4
$\$$
5

峁|

О)

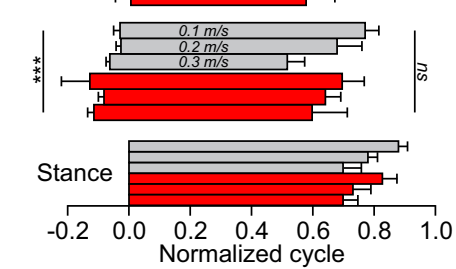

Figure 4. Speed modulation during bipedal backward locomotion before and after spinal transection. $A$, EMG activity from nine hindlimb muscles along with stance phases (horizontal thick lines) of the left (L) and right (R) hindlimbs in one cat before (BW-biped-intact) and six weeks after spinal transection (BW-biped-spinal) during backward bipedal locomotion at three speeds. Data are from Cat 5 with perineal stimulation in both directions. For muscle abbreviations, see Figure 1. $\boldsymbol{B}$, Cycle, stance and swing durations in the two states. $\boldsymbol{C}$, Stride and step lengths in the two states. $\boldsymbol{D}$, Distance at contact and liftoff in the two states. EMG burst durations $(\boldsymbol{E})$ and phasing $(\boldsymbol{F})$ in the two states. $\boldsymbol{B}-\boldsymbol{F}$, Each data point or bar is the mean \pm SD for the group ( $n=4$ cats), whereas statistical analyses were done on pooled cycles/bursts. BW-biped-intact: $n=27, n=37$, and $n=44$ cycles/bursts at $0.1,0.2$, and $0.3 \mathrm{~m} / \mathrm{s}$, respectively; BW-biped-spinal: $n=29, n=45$, and $n=32$ cycles/bursts at $0.1,0.2$, and $0.3 \mathrm{~m} / \mathrm{s}$, respectively. Asterisks indicate a significant effect of state (MLM) at $* p<0.01, * * p<0.001, * * * p<0.0001 ; \mathrm{ns}$, not significant.

consistent with control at a spinal level. Here, we compare spatiotemporal and EMG adjustments in spinal cats to an increase in treadmill speed during bipedal forward and backward locomotion to determine whether adjustments are similar in these two forms of locomotion with opposite directions (Fig. 5). Figure $5 \mathrm{~A}$ shows the EMG activity of nine selected hindlimb muscles along with the right and left hindlimb stance phases at $0.1,0.2$, 0.3 , and $0.4 \mathrm{~m} / \mathrm{s}$ in the same spinal cat (Cat 5) during forward 


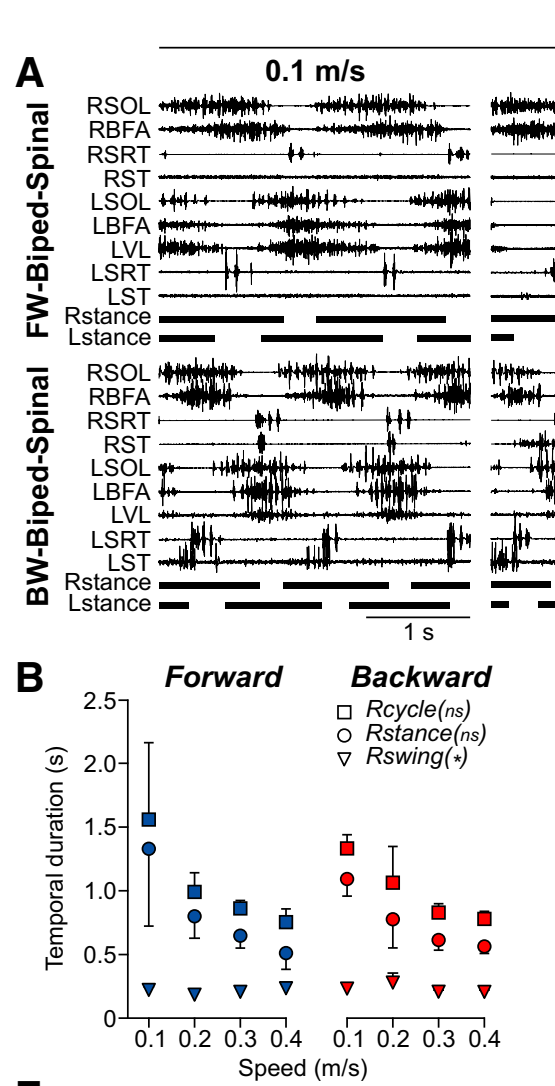

$\mathbf{E}$

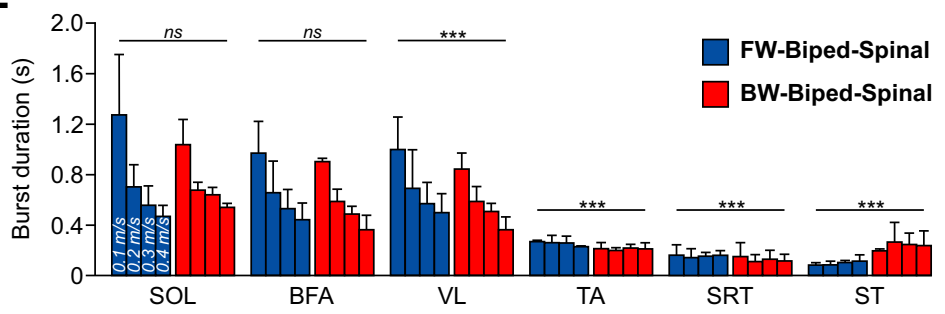

G

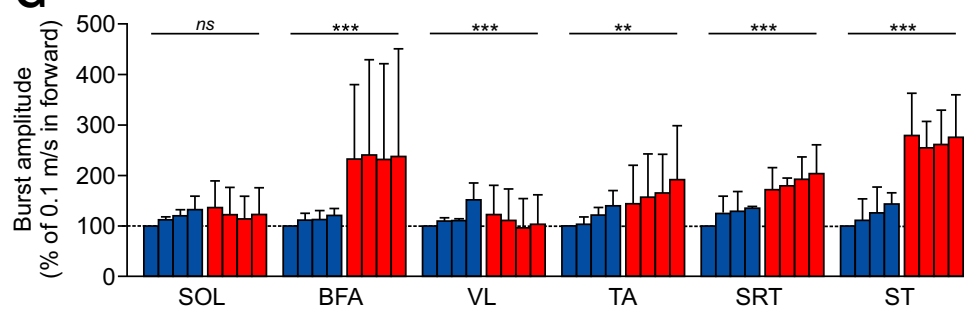

In spinal cat

-
$0.2 \mathrm{~m} / \mathrm{s}$

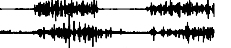

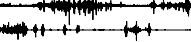

tow

$-$

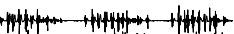
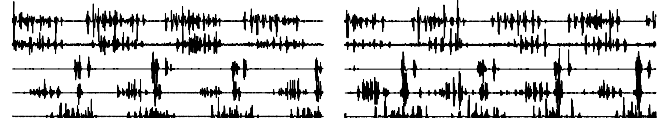

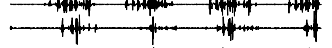
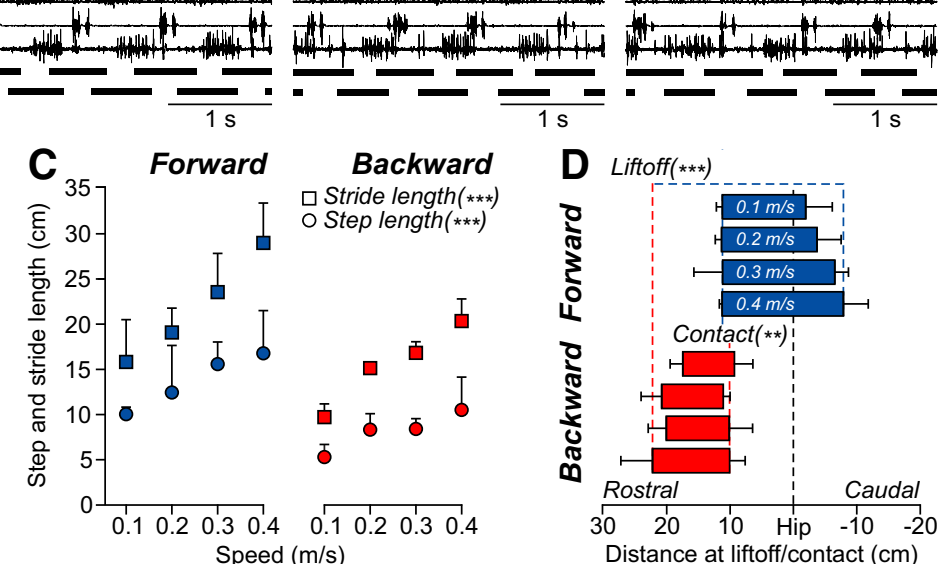

D Liftoff( $(* * x)$

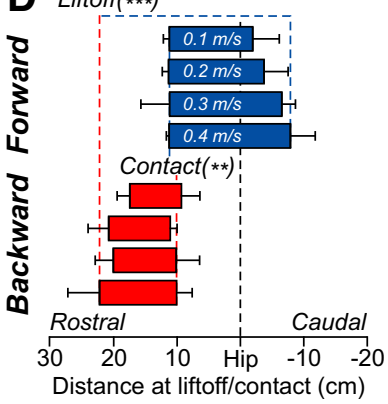

$F$
s)
5
$\frac{5}{0}$

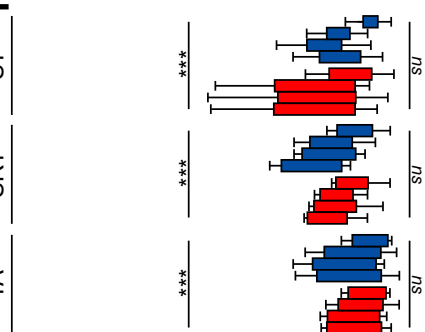

$>$

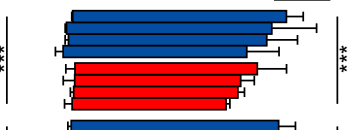

㭊|

ठิ)
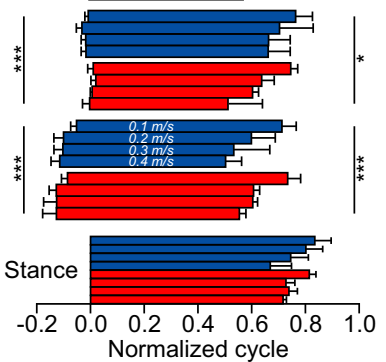

Figure 5. Speed modulation during forward and backward before and after spinal transection. $A$, EMG activity from nine hindlimb muscles along with stance phases (horizontal thick lines) of the left (L) and right (R) hindlimbs in one spinal cat six weeks after spinal transection during forward (FW-biped-spinal) and backward (BW-biped-spinal) bipedal locomotion at four speeds. Data are from Cat 5 with perineal stimulation in both directions. For muscle abbreviations, see Figure 1. B, Cycle, stance and swing durations in the two directions. C, Stride and step lengths in the two directions. $\boldsymbol{D}$, Distance at contact and liftoff in the two directions. EMG burst durations $(\boldsymbol{E})$ and phasing $(\boldsymbol{F})$ in the two directions. $\boldsymbol{B}-\boldsymbol{F}$, Each data point or bar is the mean \pm SD for the group ( $n=3$ cats), whereas statistical analyses were done on pooled cycles/bursts. FW-biped-spinal: $n=27, n=35, n=35$, and $n=35$ cycles/bursts at $0.1,0.2,0.3$, and $0.4 \mathrm{~m} / \mathrm{s}$, respectively; BW-biped-spinal: $n=25, n=38, n=33$, and $n=29$ cycles/bursts at $0.1,0.2,0.3$, and $0.4 \mathrm{~m} / \mathrm{s}$, respectively. Asterisks indicate a significant effect of direction (MLM) at $* p<0.01$, $* * p<0.001, * * * p<0.0001 ;$ ns, not significant.

and backward bipedal locomotion. In both forms of locomotion, extensor burst and stance phase durations decreased with increasing speed while flexor burst and swing durations were much less affected. We found that cycle $\left(p=9.56^{\mathrm{e}-34}\right)$ and stance $\left(p=7.55^{-41}\right)$ durations during forward and backward bipedal locomotion in spinal cats were significantly reduced by increasing treadmill speed while swing was unaffected $(p=0.113$; Fig. $5 B)$. Interestingly, cycle $(p=0.816)$ and stance $(p=0.229)$ durations did not significantly differ between forward and backward locomotion in spinal cats while swing duration was slightly 
longer during backward locomotion $(p=0.004 ; 0.02$ s or $9.0 \%$ difference).

During forward and backward locomotion in spinal cats, stride $\left(p=1.73^{\mathrm{e}-17}\right)$ and step $\left(p=7.77^{\mathrm{e}-62}\right)$ lengths significantly increased with increasing speed (Fig. $5 C$ ). When comparing the two conditions, stride $\left(p=2.34^{\mathrm{e}-31} ; 6.35 \mathrm{~cm}\right.$ or $29.1 \%$ difference) and step ( $p=3.04^{\mathrm{e}-45} ; 5.57 \mathrm{~cm}$ or $40.1 \%$ difference) lengths were, on average, significantly longer during forward locomotion. The distance of the hindpaw relative to the hip at liftoff was significantly more caudal with increasing speed during forward locomotion and more rostral during backward locomotion $(p=0.001$; Fig. $5 D)$. In contrast, the distance at contact was unaffected by speed for both forms of locomotion $(p=0.132)$. When comparing the two forms of locomotion, spinal cats executed their liftoff further from the hip during backward locomotion compared with forward locomotion ( $p=1.80^{\mathrm{e}-151} ; 15.13 \mathrm{~cm}$ or $300.8 \%$ difference) and made contact slightly further from the hip during forward locomotion ( $p=0.002 ; 1.08 \mathrm{~cm}$ or $9.6 \%$ difference).

During forward and backward locomotion in spinal cats, EMG burst durations of extensor muscles, namely SOL ( $p=$ $\left.2.61^{\mathrm{e}-52}\right)$, BFA $\left(p=6.21^{\mathrm{e}-53}\right)$, and VL $\left(p=4.44^{\mathrm{e}-41}\right)$, significantly decreased with increasing speed (Fig. $5 E$ ). On average, extensor EMG burst duration was significantly longer during forward locomotion compared with backward locomotion for VL $\left(p=2.47^{\mathrm{e}-6} ; 0.11 \mathrm{~s}\right.$ or $16.5 \%$ difference $)$ but not SOL $(p=0.926)$ or BFA $(p=0.03)$. The EMG burst duration of SRT $(p=0.001)$ and ST $(p=0.006)$ changed significantly with speed, although not consistently, whereas TA did not $(p=0.027)$. The SRT burst duration was significantly longer during forward locomotion $\left(p=2.19^{\mathrm{e}-10} ; 0.03\right.$ s or $18.2 \%$ difference) while ST burst duration was longer during backward locomotion $\left(p=9.19^{\mathrm{e}-45} ; 0.14 \mathrm{~s}\right.$ or $143.6 \%$ difference).

When looking at the phasing of EMG bursts, we found a significant shift in onsets and offsets with increasing speed for all muscles during forward and backward locomotion in spinal cats, with the exception of BFA onset (Fig. 5F). Within a normalized cycle, onsets occurred significantly earlier at faster speeds for SOL $\left(p=6.50^{\mathrm{e}-20}\right)$, VL $(p=0.003)$, TA $\left(p=3.05^{\mathrm{e}-29}\right)$, SRT $\left(p=9.17^{\mathrm{e}-49}\right)$, and ST $\left(p=8.02^{\mathrm{e}-23}\right)$ but not BFA $(p=0.04)$. On average, we observed an earlier onset during backward locomotion compared with forward locomotion for SOL $\left(p=1.16^{\mathrm{e}-13} ; 2.3 \%\right.$ difference) and ST ( $p=1.33^{\mathrm{e}-30} ; 15.1 \%$ difference) while a later onset was found for BFA $\left(p=2.65^{\mathrm{e}-19} ; 2.7 \%\right.$ difference $)$ VL $\left(p=1.40^{\mathrm{e}-10}\right.$; $2.3 \%$ difference), TA $\left(p=3.52^{\mathrm{e}-07} ; 3.2 \%\right.$ difference), and SRT $\left(p=8.17^{\mathrm{e}-17} ; 4.3 \%\right.$ difference). Offsets occurred significantly earlier at faster speeds for SOL $\left(p=9.36^{\mathrm{e}-40}\right), \operatorname{BFA}\left(p=1.81^{\mathrm{e}-31}\right), \mathrm{VL}$ $\left(p=1.62^{\mathrm{e}-13}\right)$, TA $\left(p=3.92^{\mathrm{e}-05}\right)$, SRT $\left(p=4.86^{\mathrm{e}-25}\right)$, and ST $\left(p=8.90^{\mathrm{e}-18}\right)$. On average, we observed an earlier offset during backward locomotion compared with forward locomotion for BFA $(p=0.003 ; 7.3 \%$ difference $)$ and VL $\left(p=7.03^{\mathrm{e}-18} ; 10.2 \%\right.$ difference) while it occurred later in SOL $\left(p=7.05^{\mathrm{e}-6} ; 3.8 \%\right.$ difference). We found no significant differences in offset between the two directions of locomotion for TA $(p=0.734)$, SRT $(p=$ $0.435)$, and ST $(p=0.195)$.

For mean EMG amplitude, we only found a significant increase with increasing speed for TA $\left(p=1.27^{\mathrm{e}-09}\right)$ and SRT $(p=0.001)$ but not SOL $(p=0.02)$, BFA $(p=0.861)$, VL $(p=0.04)$, and ST $(p=0.144$; Fig. $5 G)$. However, the direction of locomotion strongly affected mean EMG amplitude. It was significantly larger during backward locomotion for BFA $\left(p=1.22^{\mathrm{e}-29} ; 111.6 \%\right.$ difference), TA $\left(p=0.0003 ; 41.6 \%\right.$ difference), SRT $\left(p=1.50^{\mathrm{e}-30}\right.$; $53.3 \%$ difference), and ST ( $p=2.88^{\mathrm{e}-23} ; 122.7 \%$ difference). For $\mathrm{VL}$, it was slightly larger during forward locomotion $\left(p=3.34^{\mathrm{e}-7}\right.$;
$8.2 \%$ difference) while for SOL we observed no differences ( $p=0.595)$ between the two directions.

\section{Forward and backward locomotion in spinal cats share similar patterns of muscle activations and synergies}

How similar are patterns of muscle activations during forward and backward locomotion in spinal cats and how do they relate to joint kinematics? Figure 6 shows changes in joint angles and patterns of muscle activity during forward and backward bipedal hindlimb locomotion at $0.3 \mathrm{~m} / \mathrm{s}$ in three spinal cats. During forward and backward locomotion, the hip, knee and ankle joints flexed at swing onset followed by extension during mid- to late swing (Fig. 6A). The timing of reversal from flexion to extension varied between forward and backward locomotion as well as between cats. At stance onset, the hip extended during forward locomotion while it flexed slightly during backward locomotion. The knee joint goes through a larger excursion during backward locomotion, extending during the first half of stance, while it flexed slightly during forward locomotion. During forward and backward locomotion, the ankle sometimes showed a small flexion at stance onset, or yield, followed by an extension. Overall, these changes in joint angles were similar to the ones observed in intact cats during forward and backward locomotion, with the main differences observed at the hip and knee joints (Buford et al., 1990).

Patterns of muscle activity were similar during forward and backward locomotion in spinal cats (Fig. 6B). In the three cats, muscles active primarily in the stance phase during forward locomotion were also active during stance of backward locomotion. This was the case for the ankle extensors LG, MG, SOL, PLA, and PLO, the knee extensor VL and the hip extensors BFA, SM, GLU, and CF. Thus, during forward stance, hip extensors are active while the hip extends while during backward stance they are active while the hip flexes. At the knee, the VL is active as the knee slightly flexes during forward stance while it extends during backward stance. The pattern of activation for flexor muscles was also relatively similar during forward and backward locomotion. The ankle flexor TA displayed a prominent burst around swing onset for both directions and also some weaker activity during stance in Cats 3 and 11 (but not Cat 5), possibly to increase ankle stiffness for support. The knee flexors/hip extensors ST and BFP showed weak or inconsistent activity preceding swing onset during forward locomotion, which is typical at slow forward speeds, and strong activity at or before swing onset in the backward direction. We observed some activity during stance in Cats 5 and 11. We also observed a brief burst or a rise in activity in mid- to late-swing in ST during forward (Cat 5), backward (Cat 3) or both directions (Cat 11). The hip flexors SRT, IP, and FL were also active before and at the start of swing during forward and backward locomotion. Overall, for most muscles, the activity was larger during backward locomotion. Similar patterns of muscle activations for forward and backward locomotion were also observed in intact cats (Buford and Smith, 1990).

To determine whether forward and backward locomotion shared similar muscle synergies, we characterized muscle synergies using NNMF (Figs. 7, 8). As the number of muscle synergies for each direction was not known a priori, we first determined how much variance $\left(R^{2}\right)$ in EMG patterns was accounted for by EMG patterns reconstructed by different numbers of synergies from 1 to 10. EMG patterns reconstructed from three synergies explained over $80 \%$ of the EMG data (matrix D), specifically $92 \%$ and $91 \%$ for FW-biped-spinal and BW-biped-spinal conditions, respectively, for Cat $11 ; 85 \%$ and $83 \%$ for Cat 5 and $92 \%$ and $88 \%$ 

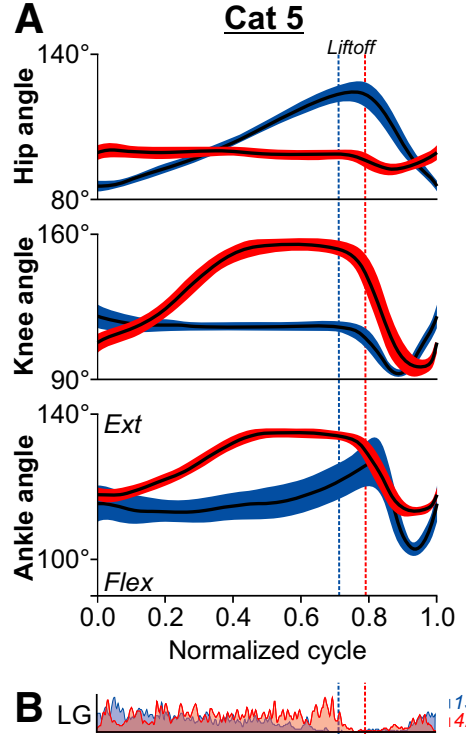

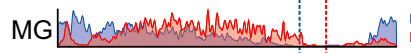

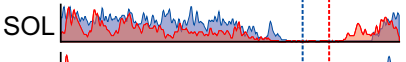

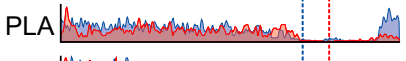

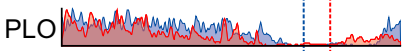
VL

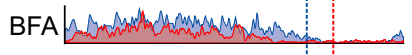

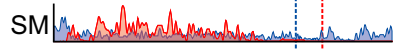

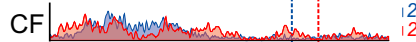
GLU \begin{tabular}{l:r}
$14.7 \mu \mathrm{V}$ \\
\hline
\end{tabular}
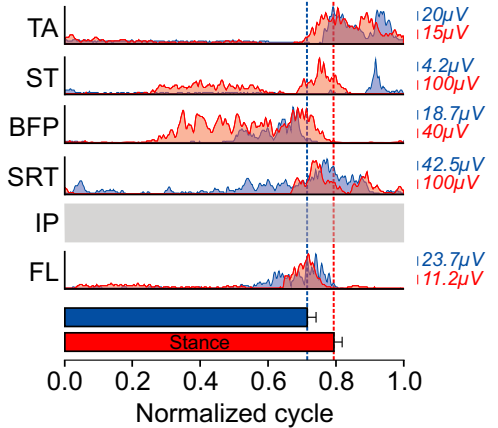

Normalized cycle
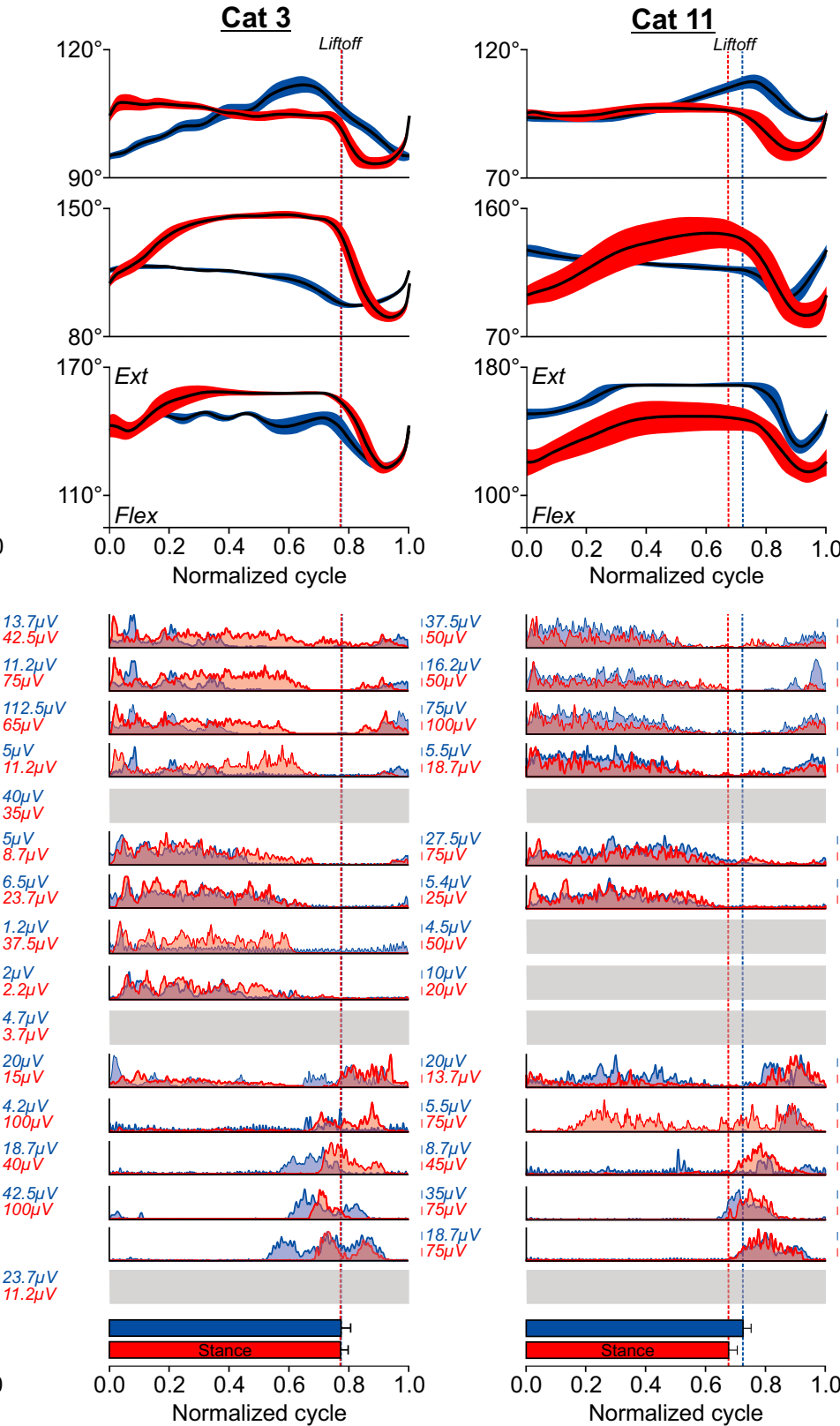

FW-Biped-Spinal BW-Biped-Spinal

Figure 6. Joint angles and normalized muscle activations during forward and backward locomotion in spinal cats. $\boldsymbol{A}$, Angles of the hip, knee, ankle joints during locomotor cycle normalized to stance onset in three spinal cats. Thick black lines represent averaged cycles while the area indicate standard deviations. $\boldsymbol{B}$, Averaged rectified muscle activity during locomotor cycle normalized to stance onset for the following muscles grouped by main biomechanical function: LG, lateral gastrocnemius; MG, medial gastrocnemius; SOL, soleus; PLA, plantaris; PL0, peroneus longus; VL, vastus lateralis; BFA, biceps femoris anterior; SM, semimembranosus; CF, caudofemoralis; GLU, gluteus maximus; TA, tibialis anterior; ST, semitendinosus; BFP, biceps femoris posterior; SRT, anterior sartorius; IP, iliopsoas; FL, tensor fascia latae. In $\boldsymbol{A}, \boldsymbol{B}$, joint angles and normalized EMG bursts are the average of 10-12 cycles/bursts per cat in both directions. Vertical dashed lines indicate paw liftoffs or swing onset in the two directions.

for Cat 3. The corresponding $R^{2}$ ranges between individual cats for 4,5 , and 6 synergies were $92-96 \%$ and $88-95 \%, 95-98 \%$ and $93-97 \%$, and $97-99 \%$ and $96-98 \%$, respectively, for FW-bipedspinal and BW-biped-spinal locomotion. Because the exact number of muscle synergies for accurate EMG data reconstruction without inclusion of noise cannot be determined with absolute certainty (Cheung et al., 2005), we arbitrarily selected the range of $R^{2}$ between $93 \%$ and $98 \%$ for an appropriate number of synergies (i.e., 5) that captured most of the EMG variance with little noise.

The first three muscle synergies, characterized by the muscle weights $\mathrm{W}_{1}, \mathrm{~W}_{2}$, and $\mathrm{W}_{3}$ (Fig. $7 \mathrm{~A}$ ), were generated mostly by extensors in each cat (first 10 muscles on $x$-axis for Cat 5 ) for forward and backward locomotion. There was one exception for forward locomotion of Cat 3, its extensors did not contribute to synergy 3 . These synergies were active during the stance phase with synergy 1 being most active at the swing-to-stance transition (Fig. $7 B, C_{1}-C_{3}$ ). Synergy 3 of forward locomotion in Cat 3 was active at the stance-to-swing transition. Synergies 4 and 5 mainly consisted of flexors (subsequent muscles on $x$-axis) with the exception of SOL and PLA (Cat 11), LG, PL and BFA (Cat 5), and LG and SOL (Cat 3), mostly in backward locomotion. These synergies were active at the stance-to-swing transition (synergy 4) and during the swing phase (synergy 5). The MLM analysis revealed significant differences in synergy weights of most 
A
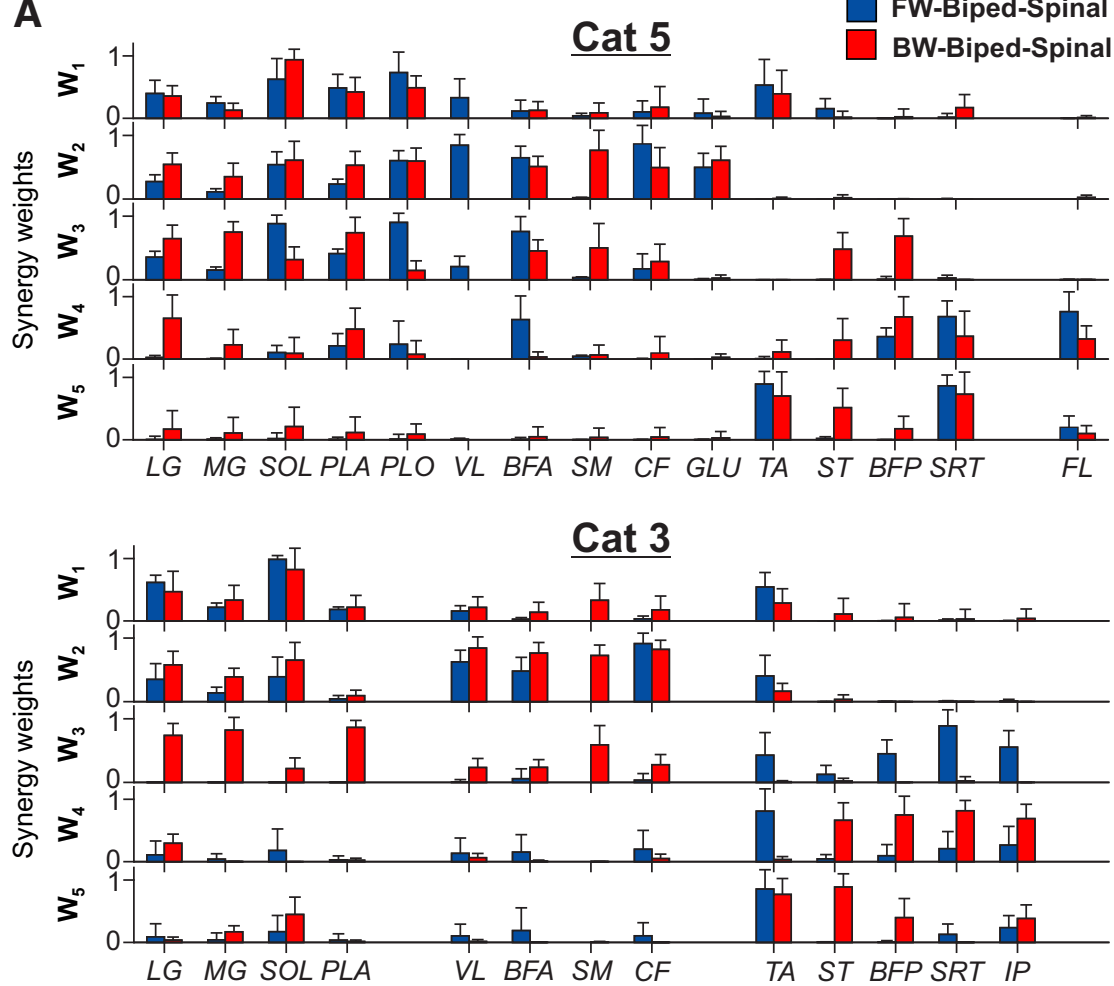

Cat 11

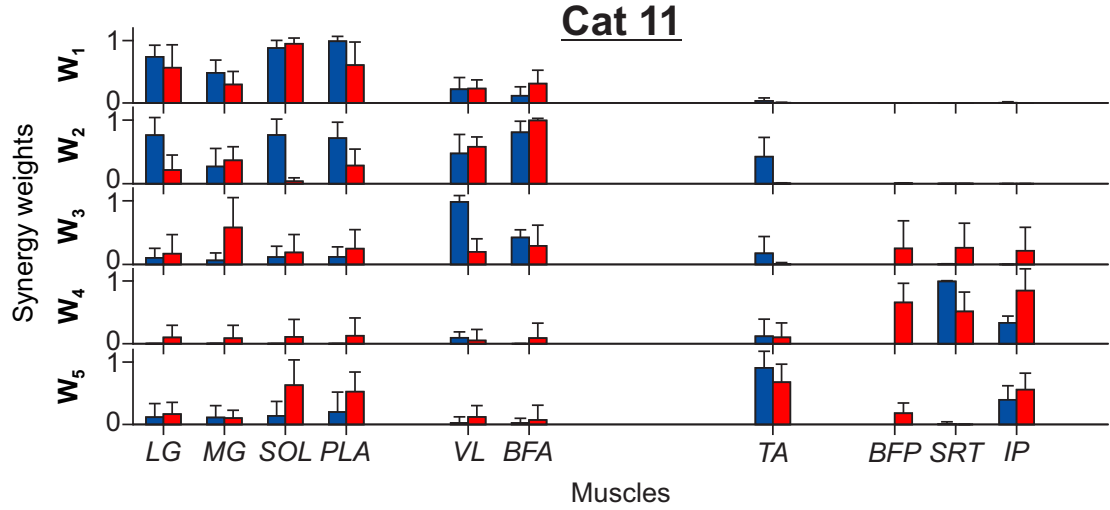

B
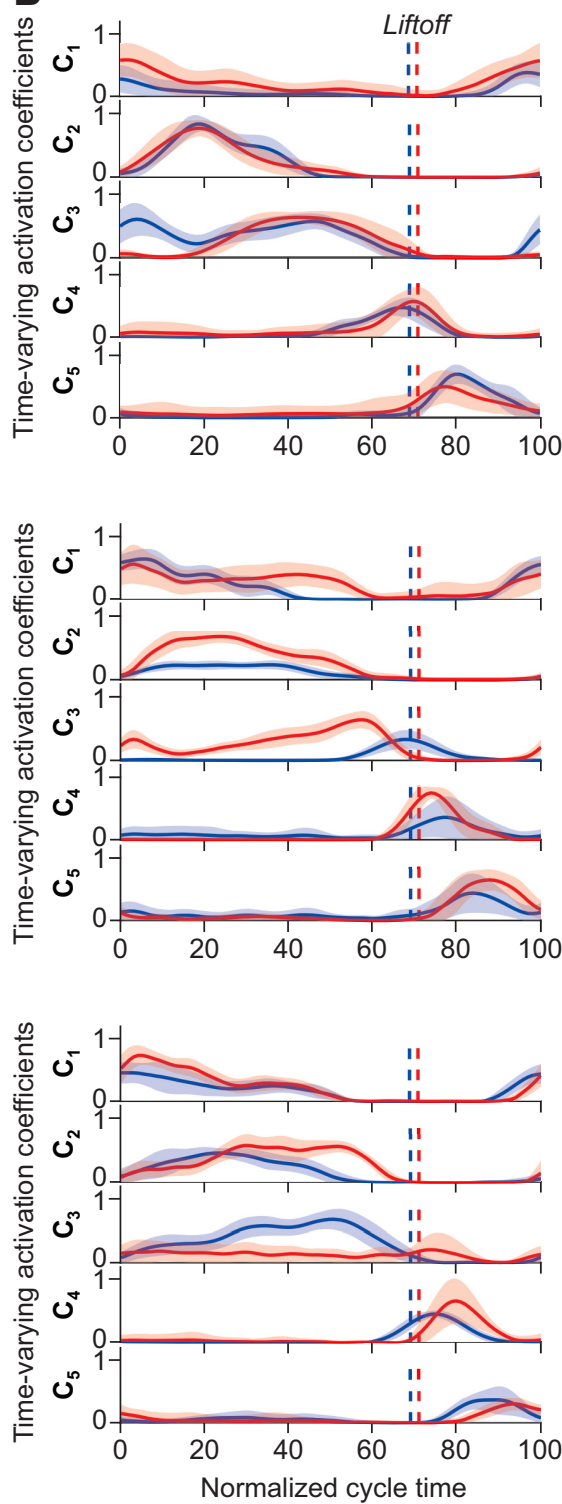

Figure 7. Five muscle synergies and their time-varying activation patterns extracted during forward and backward locomotion in three spinal cats at $0.3 \mathrm{~m} / \mathrm{s}$. $A$, Synergy weights (mean \pm SD) in five synergies $\left(W_{1}-W_{5}\right)$ in the forward and backward locomotor conditions, where a value of 1 indicates maximal weight. The means and SDs are from 20 extractions of muscle synergies for each cycle, direction and cat (Cat 5: 8 forward and 14 backward cycles; Cat 3: 11 forward and 6 backward cycles; and Cat 11: 9 forward and 4 backward cycles). For muscle abbreviations, see Figure 6 legend. Note a different number of muscles is included in synergy analysis for the three cats. In addition, BFP activity of forward locomotion in Cat 11, VL activity of backward locomotion in Cat 5 and SM activity of forward locomotion in Cat 3 are not included in synergy analysis. $B$, Time-varying activation coefficients $\left(C_{1}-C_{5}\right)$ for the five synergies in the forward and backward locomotion as a function of the normalized cycle duration ( 0.0 corresponds to stance onset) for three cats. A value of 1 indicates a maximal activation within the normalized cycle. The thick lines and surrounding area represent the mean and SD, respectively. Vertical dashed lines indicate swing onsets.

extensor muscles in synergies 1-3 of each cat between forward and backward locomotion (range $p=2.19^{\mathrm{e}-278}$ to $p=0.001$ ). Depending on the cat, weights of the same extensor muscles were often higher in forward locomotion (e.g., LG, Cat 11, synergy 2, $p=4.55^{\mathrm{e}-101}$; SOL, Cat 11 , synergy $2, p=4.61^{\mathrm{e}-176}$; BFA, Cat 5 , synergy $2, p=2.66^{\mathrm{e}-11}$ ) or backward locomotion (SOL, synergy 2, Cats 5 and $3, p=0.001$ and $p=1.54^{\mathrm{e}-33}$; LG, Cats 5 and 3 , synergy $2, p=1.09^{\mathrm{e}-38}$ and $p=6.11^{\mathrm{e}-26} ; \mathrm{BFA}$, Cat 3 , synergy 2 , $p=3.66^{\mathrm{e}-39}$ ) or no differences were detected between directions (LG, Cat 5, synergy 1, $p=0.045$; VL, Cat 11 , synergy $1, p=0.666$; $\mathrm{BFA}$, Cat 5 , synergy $1, p=0.557)$. In flexor synergies 4 and 5 , we found that synergy weights of flexors TA and FL were higher in forward locomotion $\left(p=2.19^{\mathrm{e}-278}-7.00^{\mathrm{e}-06}\right)$, and the weights of IP were higher in backward locomotion $\left(p=4.73^{\mathrm{e}-90}\right.$ to $\left.2.22^{\mathrm{e}-10}\right)$ in most recorded cats. The knee flexor/hip extensor ST and BFP had higher weights in synergies four of backward locomotion $\left(p=1.40^{\mathrm{e}-198}\right.$ to $\left.1.33^{\mathrm{e}-47}\right)$, whereas SRT had higher weights in forward locomotion of Cats 11 and $5\left(p=2.16^{\mathrm{e}-78}\right.$ to $\left.5.61^{\mathrm{e}-09}\right)$ and backward locomotion of Cat 3 (synergy $4, p=2.61^{\mathrm{e}-172}$; Fig. $7 A$ ).

The MLM analysis applied to the common and direction-specific synergies obtained from the set of EMG patterns of 7 muscles recorded in all cats and directions (forward, backward, combined) demonstrated that the factors "cat" and "direction" significantly affected synergy weights across synergies and muscles $\left(p=5.20^{\mathrm{e}-05}\right.$ and $p=1.45^{\mathrm{e}-47}$, respectively; Fig. $\left.8 A\right)$. Pairwise comparisons revealed that the only non-significant effect of the factor cat on muscles in extensor synergies 1-3 was for BFA (synergy 1, combined direction, $p=0.019$ ) and MG (synergy 3, forward 
A
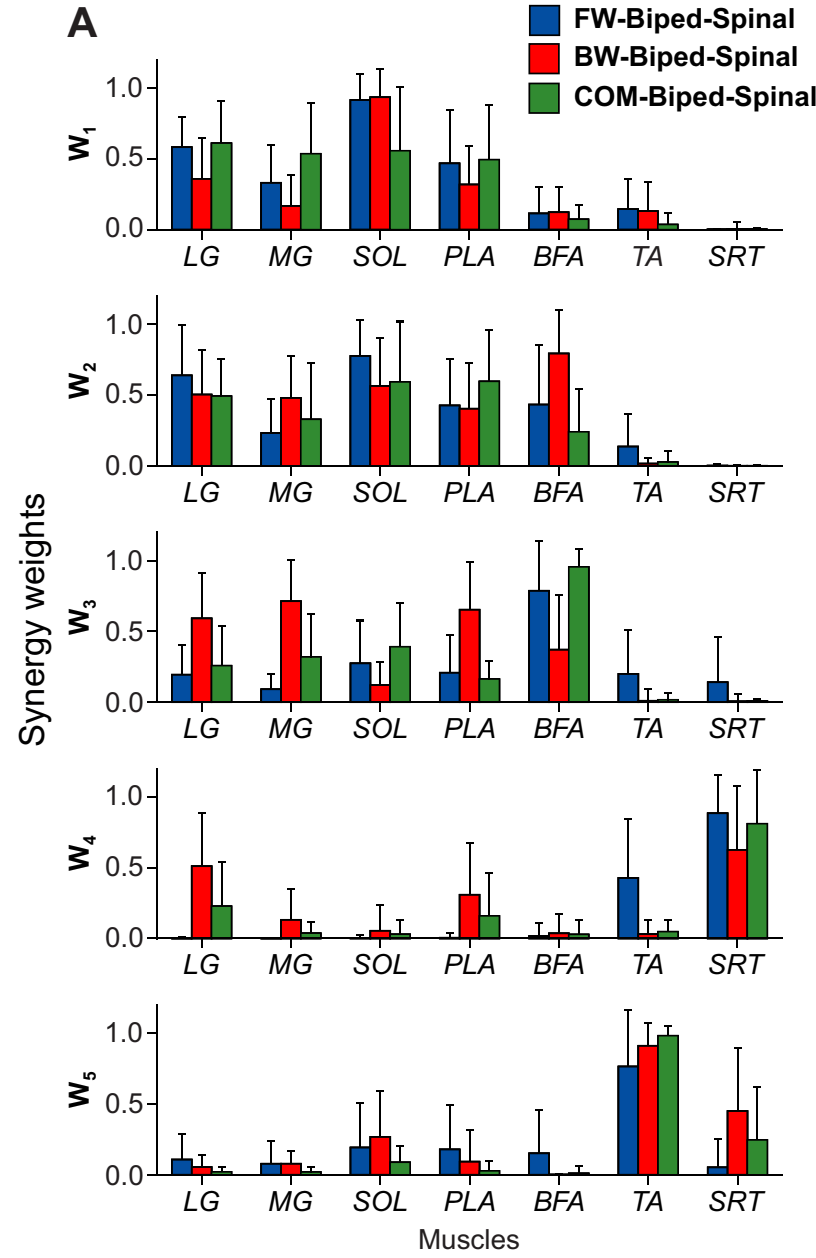

B FW-Biped-Spinal BW-Biped-Spinal
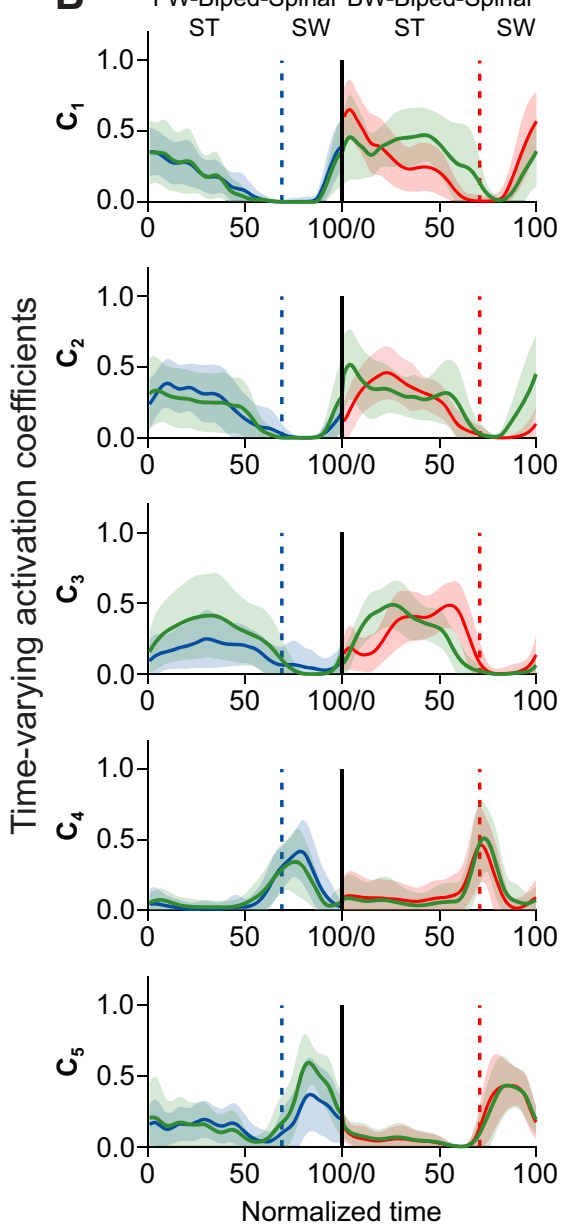

C
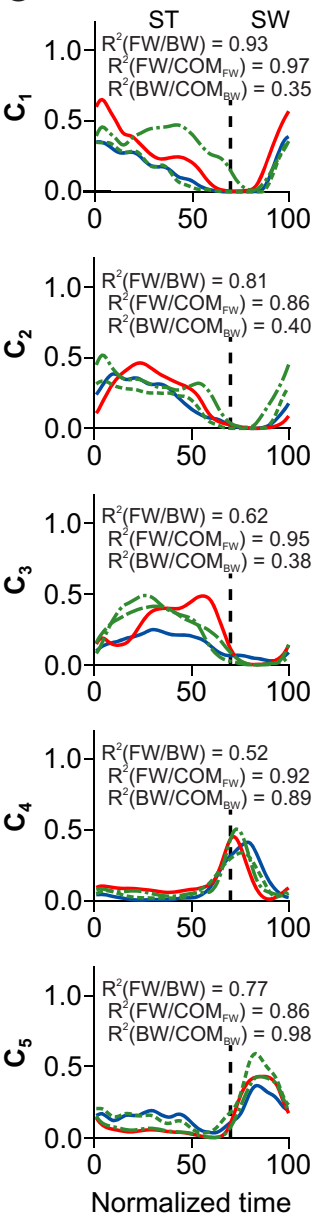

Figure 8. Five muscle synergies and their time-varying activation patterns extracted during forward, backward and combined locomotor conditions in three spinal cats together at $0.3 \mathrm{~m} / \mathrm{s}$. $A$, Synergy weights (mean $\pm S D$ ) in five synergies $\left(W_{1}-W_{5}\right)$ in the forward, backward and combined locomotor conditions, where a value of 1 indicates a maximal weight. The means and SDs are from 20 extractions of muscle synergies for each cycle, direction and cat (Cat 11, 4 forward and backward cycles; Cat 5, 8 forward and backward cycles; and Cat 3, 6 forward and backward cycles). For muscle abbreviations, see Figure 6 legend. $\boldsymbol{B}$, Time-varying activation coefficients $\left(C_{1}-C_{5}\right)$ for the five synergies extracted from forward EMG patterns (blue lines to the left from the vertical black line), from backward EMG patterns (red lines to the right of the black vertical line) and from combined forward-backward EMG patterns (green lines). The horizontal axis represents the normalized cycle times of forward (before the black vertical line) and backward locomotion (after the black line); 0.0 corresponds to stance onset. A value of 1 indicates a maximal activation within the normalized cycle. The thick lines and surrounding area represent the mean and SD, respectively. Vertical dashed lines separate stance (ST) and swing (SW) phases of forward and backward locomotion. $\boldsymbol{C}$, The same time-varying activation coefficients $\left(C_{1}-C_{5}\right)$ as in $\boldsymbol{B}$ plotted as a function of the normalized cycle time. Blue and red lines denote forward and backward locomotion, green dashed and dashed with dot lines denote the forward and backward components of the combined matrix of activation coefficients $\left(\mathcal{C}_{\text {Com_fw }}\right.$ and $C_{\text {COM_BW, }}$ respectively). Black dashed line corresponds to the mean relative time of swing onset in forward and backward locomotion. Coefficients $\mathrm{R}^{2}$ computed between activation coefficients of forward locomotion (FW), backward locomotion (BW), and forward and backward components of the combined forward and backward locomotion ( $\mathrm{COM}_{\mathrm{FW}}$ and $\left(\mathrm{CO}_{\mathrm{BW}}\right)$.

direction, $p=0.392$ ), and for TA in synergy 4 (backward, $p=0.969$, and combined directions, $p=0.095$ ) and in synergy 5 (combined direction, $p=0.165$ ). Similarly, there was a non-significant effect of direction on LG in synergy 1 of Cat $3(p=0.510)$ and in the SRT in synergies 4 and 5 in Cat $11(p=1.000)$. Synergy analysis across three cats and both directions was consistent with that of individual cats (Fig. 7A), synergies 1 through three consisted of pure extensor muscles (SOL and BFA) and two-joint muscles active during the stance phase (LG, MG, and PLA), whereas synergies 4 and 5 included the flexors TA and SRT active during the swing phase (Figs. 7, 8).

We found generally similar results for synergies (synergy weights) spanning both forward and backward locomotion (compare the green bars corresponding to the combined forward-backward condition with the blue and red bars of the forward and backward locomotion; see Fig. 8A). The time-varying activation coefficients for all five synergies generally had similar patterns between forward and backward locomotion in individual cats (with the exception of synergy 3; Fig. $7 B$ ) and for all cats combined (Fig. 8B), including activation coefficients of matrix $\mathbf{C}_{\mathrm{COM}}$ containing both forward $\mathbf{C}_{\mathrm{COM} \_\mathrm{FW}}$ and backward $\mathrm{C}_{\mathrm{COM} \_\mathrm{BW}}$ components (Fig. 8B,C; Table 1). The time-varying patterns of muscle synergies spanning both forward and backward locomotion, green lines, were generally changing in phase with forward (blue) and backward (red) lines and had higher values during the stance phase for synergies $1-3$ and during the swing phase for synergies 4 and 5 (Fig. $8 B, C$ ). The $R^{2}$ calculated between each of the mean time-varying patterns were typically between 0.52 and 0.98 , with three exceptions $\left(R^{2}=0.35-0.40\right.$, BW/COM ${ }_{\mathrm{BW}}$, synergies 1-3; Fig. $8 \mathrm{C}$ ).

Despite qualitatively similar activation patterns of synergies for forward and backward locomotion, as well as of the forward and backward components of activation patterns of synergies spanning both directions (Fig. 8B,C), the three-way pairwise 


\section{Backward locomotion on split-belt treadmill}

A

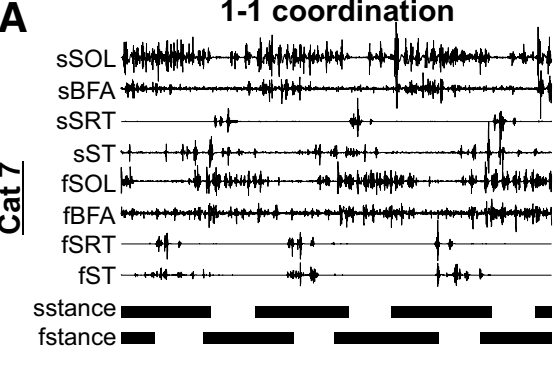

2-1 coordination
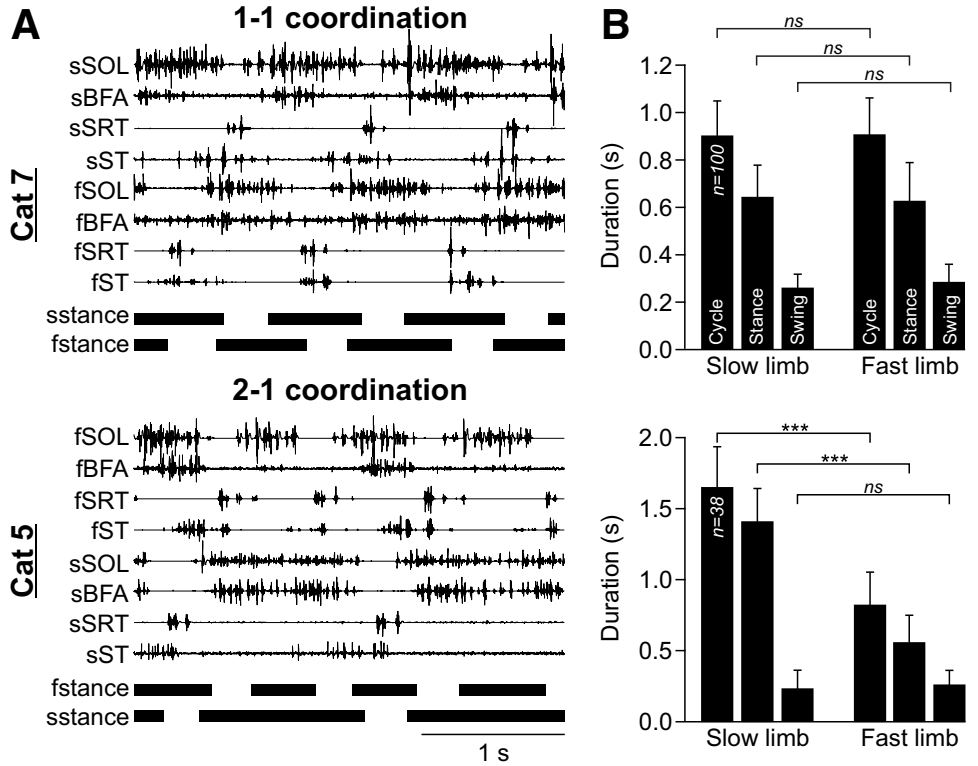

Figure 9. Backward locomotion on a split-belt treadmill in spinal cats. A, EMG activity from eight hindlimb muscles along with stance phases (horizontal thick lines) of the left (L) and right (R) hindlimbs in two spinal cats with the slow (s) hindlimb stepping at $0.1 \mathrm{~m} / \mathrm{s}$ and the fast (f) hindlimb stepping at $0.3 \mathrm{~m} / \mathrm{s}$. For muscle abbreviations, see Figure 6 legend. $\boldsymbol{B}$, Cycle, stance and swing durations (mean \pm SD) of the slow and fast hindlimbs with 1-1 coordination ( $n=100$ cycles) and $2-1$ coordination ( $n=38$ cycles) patterns (pooled data from Cats 5, 7, and 11). Asterisks indicate a significant difference between the slow and fast limbs (paired $t$ test) at $* * * p<0.0001 ; \mathrm{ns}$, not significant.

analyses of the activation patterns revealed significant effects of locomotor direction (range $p=6.27^{\mathrm{e}-256}$ to $p=0.002$ ), as well as binned cycle time (range $p=4.59^{\mathrm{e}-307}$ to $p=0.007$ ) and cat (range $p=2.38^{\mathrm{e}-254}$ to $\left.p=3.93^{\mathrm{e}-10}\right)$. Pairwise comparisons revealed few instances where activation coefficients of extensor synergies 1-3 were not significantly different between directions during the stance phase, in time bin 4 (between forward direction and backward component of the combined directions, $p=0.938$ ) and bin 6 (between forward direction and forward component of the combined directions, $p=0.899$ ) of synergy 2; in time bins 1 (between backward direction and forward and backward components of the combined directions, $p=0.012$ to $p=0.278$ ) and 3 (forward and backward directions, $p=0.024$ ) of synergy 3. Activation coefficients of flexor synergies 4 and 5 likewise had few non-significant differences during the swing phase between directions, in time bin 7 (between forward, backward and backward component of combined directions, $p=0.013$ to $p=0.902$ ) and bin 10 (between backward and both components of the combined directions, $p=0.300$ to $p=0.953$ ) in synergy 4 ; and time bins 7,9 , and 10 (between backward and backward component of the combined directions, $p=0.104-0.952$ ) and bin 8 (between backward and forward and between backward and backward component of the combined directions, $p=0.041$ and $p=0.014$, respectively) in synergy 5 . Despite significant differences in activation coefficients between directions, the root mean square differences were relatively small, i.e., between 0.023 (forward direction vs forward component of the combined directions in synergy 1) and 0.210 (forward and backward components of the combined directions in synergy 1 ) across all five synergies (Fig. $8 B, C$ ). Thus, the above analysis of EMG activity patterns of multiple hindlimb muscles during forward and backward locomotion in spinal cats demonstrated that these patterns are accurately described by a small number of muscle synergies (five synergies) with their activation patterns.
We found that EMG patterns reconstructed from five synergies spanning both forward and backward locomotion (the synergy weights $\mathbf{W}_{\mathrm{COM}}$ and their time-dependent forward $\left(\mathbf{C}_{\mathrm{COM} \_\mathrm{FW}}\right)$ and backward components of activation coefficients $\mathrm{C}_{\mathrm{COM} \_\mathrm{BW}}$ ), as well as various combinations of synergy weights $\left(\mathbf{W}_{\mathrm{FW}}\right.$ and $\left.\mathbf{W}_{\mathrm{BW}}\right)$ and the activation patterns of forward and backward locomotion $\left(\mathrm{C}_{\mathrm{FW}}\right.$ and $\mathrm{C}_{\mathrm{BW}}$ ) accurately reproduced the recorded EMG patterns of forward locomotion, i.e., matrix $\mathbf{D}_{\mathrm{FW}}$ $\left(R^{2}=0.687-0.947, \mathrm{RMSD}=0.108-0.051\right)$ and of backward locomotion ( $\mathbf{D}_{\mathrm{BW}} ; R^{2}=0.593-0.967$, RMSD $=0.149-0.056$; Table 1). Overall, the common synergies spanning both directions and their corresponding activation patterns $\left(\mathbf{W}_{\mathrm{COM}} \mathbf{C}_{\mathrm{COM} \_\mathrm{FW}}\right.$ and $\mathbf{W}_{\mathrm{COM}} \mathbf{C}_{\mathrm{COM} \_\mathrm{BW}}$ ) captured statistically indistinguishable levels of variance compared with that of either the forward or backward synergies with their activation patterns $\left(\mathrm{W}_{\mathrm{FW}} \mathrm{C}_{\mathrm{FW}}\right.$ and $\mathbf{W}_{\mathrm{BW}} \mathrm{C}_{\mathrm{BW}}$, respectively; Table 1). Taken together, the muscle synergy analysis revealed that forward and backward locomotion in spinal cats shared three extensor and two flexor synergies (i.e., synergy weights). The time-varying activation patterns of all five synergies were also shared between forward and backward locomotion, as these patterns in combination with the synergy weights of either forward or backward locomotion typically accounted for $\sim 70 \%$ of EMG variance (Table 1).

Backward locomotion adjusts to different speeds for the left and right hindlimbs on a split-belt treadmill in spinal cats A few studies have shown that spinal cats adjust to different speeds for the left and right hindlimbs when stepping on a splitbelt treadmill, indicating a control at the level of spinal sensorimotor circuits (Forssberg et al., 1980; Frigon et al., 2013, 2017). Similar findings have been reported in intact (Halbertsma, 1983; Frigon et al., 2015) and decerebrate (Kulagin and Shik, 1970) cats, as well as healthy humans (Dietz et al., 1994; Prokop et al., 1995; Jensen et al., 1998; Reisman et al., 2005; Choi and Bastian, 2007; Finley et al., 2013). To maintain an equal number of steps between the slow and fast sides, or a 1:1 coordination, the stance phase of the slow limb and the swing phase of the fast limb are prolonged. In intact and spinal cats, when the difference in speeds between the two belts is increased, the number of steps taken between the fast and slow differs, generating 2:1, 3:1, 4:1, and 5:1 coordination patterns (Forssberg et al., 1980; Frigon et al., 2017). Here, we show that spinal cats also adjust to a splitbelt treadmill during backward locomotion (Fig. 9). Figure 9 shows the EMG activity of eight to nine selected hindlimb muscles along with the stance phases of the fast and slow limbs stepping at 0.3 and $0.1 \mathrm{~m} / \mathrm{s}$, respectively, in two spinal cats. To adjust to backward split-belt locomotion, cats maintained a 1:1 coordination or adopted a 2:1 coordination (Fig. 9A). To determine temporal adjustments during backward locomotion in spinal cats, we pooled all cycles from three cats (Cats 5, 7, and 11) and separated those with a 1:1 or 2:1 coordination between the fast and slow limbs for statistical analysis (Fig. 9B). When cats maintained a 1:1 coordination, we found no significant differences in cycle duration between the slow and fast sides. We also found no significant differences in stance duration between the 
slow and fast sides, although there was a trend for the swing phase to be longer in the fast limb. With a 2:1 coordination, cycle and stance durations were significantly smaller in the fast limb while swing duration was not significantly different between limbs.

\section{Discussion}

\section{Backward locomotion in spinal cats requires increased} excitability within spinal sensorimotor circuits

All but one of our spinal cats generated backward locomotion. However, all cats required perineal stimulation, indicating that backward locomotion necessitates increased excitability within spinal neuronal circuits. Two studies reported that spinal cats could not perform backward locomotion, although they stepped forward with weight support (Robinson and Goldberger, 1986; Buford and Smith, 1990). Barbeau et al. (1987) showed backward locomotion in one spinal cat treated with clonidine, an $\alpha-2$ noradrenergic agonist known to facilitate hindlimb locomotion in spinal cats (Rossignol et al., 1998). Courtine et al. (2009) showed that spinal rats treated with serotonergic agonists and receiving electrical epidural stimulation of the spinal cord performed backward locomotion. These studies did not mention if they attempted backward locomotion with perineal stimulation only. Taken together, backward locomotion in spinal animals requires greater spinal neuronal excitability, contrary to forward locomotion, which recovers without exogenous factors (Bélanger et al., 1996; Lovely et al., 1990; Harnie et al., 2019). We propose that supraspinal pathways normally elevate spinal neuronal excitability required for backward locomotion, such as those releasing monoamines. Perineal stimulation, monoamines and spinal epidural electrical stimulation appear to facilitate hindlimb locomotion through a similar mechanism, by increasing spinal locomotor CPG excitability and/or neurons downstream, as suggested in humans with spinal cord injury for forward walking (Hofstoetter et al., 2017).

Harnie et al. (2019) recently showed that recovery of standing and forward locomotion does not require task-specific training. We extend these results by demonstrating that recovery of backward locomotion also does not require task-specific training, as none of our cats were trained to perform backward locomotion after spinal transection. Thus, the neuronal circuitry for backward locomotion is contained within the spinal cord. Interestingly, some animals could only step in one direction, either forward (Cat 6) or backward (Cats 7 and 8). In Cat 6 , forward locomotion was robust without perineal stimulation and moderate to strong perineal stimulation disrupted the pattern, producing hyperflexions and stomping (Harnie et al., 2019). In Cats 7 and 8, although perineal stimulation increased rhythmic activity in the forward direction, the hindlimbs could not move rostrally during swing. However, when we reversed the treadmill, these animals performed full weight bearing backward locomotion. We propose that forward and backward locomotion require optimal levels of excitability within spinal sensorimotor circuits. Below a threshold, the spinal CPG and its interactions with limb sensory feedback cannot produce forward or backward locomotion, whereas above a certain level, increased excitability disrupts sensorimotor interactions and locomotion. As sensory cues for forward and backward locomotion differ, some animals can only step in one direction.

Indeed, our results clearly show that sensory feedback from the limbs selects the appropriate direction, with no other directional signal available. The most likely sensory cue is stretch feedback from hip muscles. When the treadmill belt moves backward, the hip extends and at a certain angle triggers the stance-to-swing transition for forward locomotion (Grillner and Rossignol, 1978), probably through stretch-sensitive afferent inputs of hip flexor muscles (Andersson and Grillner, 1983). When the belt moves forward, the hip flexes, stretching hip extensors, initiating the stance-to-swing transition for backward locomotion. The hindpaws of spinal cats at the stance-toswing transition for backward locomotion were more rostral compared with intact cats (Fig. 3). In other words, swing onset required greater hip flexion or hip extensor muscle stretch, consistent with greater reliance on sensory feedback in spinal animals to regulate phase transitions (Rossignol and Frigon, 2011). Perineal stimulation facilitated backward locomotion if initiated while spinal cats were standing. If the hindlimbs remained stuck rostrally, we stopped the treadmill belt, replaced the animal in a standing position and restarted the trial. Increasing spinal neuronal excitability with perineal stimulation makes the spinal locomotor CPG more responsive to sensory feedback from the limbs. Although we favor hip muscle afferents in selecting movement direction and regulating phase transitions, we cannot exclude contributions from other afferents, including load receptors in extensor muscles or skin (Duysens and Pearson, 1980; Duysens et al., 2000; Rossignol et al., 2006; Pearson, 2008). It will be important to determine the specific sources of sensory feedback that facilitate forward and backward locomotion and how these interact with weakened central locomotor networks, which could have important implications for locomotor rehabilitation after spinal cord injury in humans.

\section{Spinal sensorimotor circuits adjust to task demands during backward locomotion}

Spatiotemporal and EMG adjustments to increasing speed during backward locomotion in spinal cats resembled those observed in intact cats (Figs. 3, 4), indicating a spinal control. The most notable difference between the intact and spinal states was the more rostral placement of the paw at liftoff and contact in spinal cats. As stated, the more rostral position of the paw at liftoff likely increases feedback from stretch-related afferents of hip extensor muscles to facilitate the stance-to-swing transition as compensation for reduced spinal neuronal excitability in spinal cats.

It is well established that cycle and stance durations decrease with increasing speed while the swing phase changes much less during forward locomotion, as observed in intact and spinal animals, consistent with a spinal control (for review, see Gossard et al., 2011). Intact cats (Buford et al., 1990) and human infants (Lamb and Yang, 2000) also reduced cycle and stance durations concurrently during backward locomotion with increasing speed while swing duration remained relatively unchanged. We extend this to backward locomotion in spinal cats. Therefore, spinal sensorimotor circuits control the strategy to reduce cycle duration by changing phase proportions during forward and backward locomotion with increasing speed.

Stride and step lengths became longer with increasing speed during forward and backward locomotion in intact and spinal cats (Figs. 3, 4), as reported during forward locomotion in intact and spinal cats (Dambreville et al., 2015), and backward locomotion in intact cats (Buford et al., 1990). In both directions, longer stride and step lengths resulted from greater hindpaw displacement at the stance-to-swing transition, more caudal and rostral for forward and backward locomotion, respectively. Paw placement at contact during forward and backward locomotion remained invariant. Some have proposed that proper paw 
contact position is crucial for dynamic balance (Halbertsma, 1983; Klishko et al., 2014). Therefore, spinal sensorimotor circuits control spatial adjustments to speed during backward locomotion.

Split-belt locomotion requires adjustments in cycle and phase durations (Kulagin and Shik, 1970; Forssberg et al., 1980; Halbertsma, 1983; Dietz et al., 1994). During forward split-belt locomotion, to maintain 1:1 coordination in the number of steps taken by slow and fast limbs, stance and swing durations of the slow and fast limbs, respectively, are proportionally prolonged, as shown in intact and spinal cats (Forssberg et al., 1980; Halbertsma, 1983; Frigon et al., 2013, 2017), and humans (Dietz et al., 1994). Spinal cats also performed split-belt backward locomotion, albeit not necessarily by adjusting stance and swing phase proportions bilaterally (Fig. 9). This could be because of the small difference in speed $(0.2 \mathrm{~m} / \mathrm{s})$ between slow and fast limbs. Spinal cats also produced 2:1 or 3:1 coordination patterns between fast and slow limbs during backward split-belt locomotion, as shown in intact and spinal cats during forward split-belt locomotion (Forssberg et al., 1980; Halbertsma, 1983; Kuczynski et al., 2017; Frigon et al., 2017). These results highlight the flexibility of spinal networks controlling forward and backward locomotion and their regulation by asymmetric sensory feedback from the limbs.

\section{A shared spinal network for forward and backward locomotion?}

Is the spinal locomotor CPG for forward and backward locomotion shared for both directions? As discussed, we observed similar spatiotemporal strategies for adjusting to increasing speed for forward and backward locomotion. We also found similar patterns of muscle activations and synergies in forward and backward directions in spinal cats, as shown in intact cats (Buford and Smith, 1990), human adults (Winter et al., 1989), and human infants (Lamb and Yang, 2000). These observations are consistent with a shared spinal locomotor network. The release of neuromodulators, supraspinal inputs or somatosensory feedback likely reconfigure the spinal network to generate different locomotor behaviors and directions, as shown in different vertebrates (Matsushima and Grillner, 1992; Li et al., 2007; Berkowitz, 2008; Liao and Fetcho, 2008; Frigon, 2009).

It was proposed that the spinal locomotor network produces a vertical component (VC) and a horizontal component $(\mathrm{HC})$ to generate locomotion in different directions (Musienko et al., 2012; Deliagina et al., 2019). Individual spinal interneurons located at L5-L6 discharged in both locomotor directions while others discharged in one direction only (Deliagina et al., 2019). The VC and $\mathrm{HC}$ were respectively suggested to correspond to the rhythm-generating and pattern formation levels of a twolevel spinal locomotor CPG (McCrea and Rybak, 2008). How do our synergies fit into proposed CPG schemes? Within the framework of a two-level CPG, the pattern formation level might contain interneuronal populations underlying common muscle synergies that engage in different motor behaviors (Hart and Giszter, 2010; Takei et al., 2017; Yang et al., 2019), like our $\mathbf{W}_{\mathrm{COM}}$ spanning both forward and backward locomotion. These pattern formation interneuronal populations might receive timevarying behavior-specific inputs (e.g., $\mathbf{C}_{\mathrm{FW}}$ and $\mathrm{C}_{\mathrm{BW}}$ ) from the rhythm generator level shaped by somatosensory feedback during the flexor and extensor phases. Alternatively, the pattern formation might contain behavior-specific synergies (e.g., $\mathbf{W}_{\mathrm{FW}}$ and $\mathbf{W}_{\mathrm{BW}}$ ) that are selected for a given behavior by somatosensory feedback and that receive a common time-varying input $\left(\mathbf{C}_{\mathrm{COM}}\right)$. Our statistical analysis of muscle synergies and their activation patterns during forward and backward locomotion does not allow us to distinguish between the two possibilities, as the muscle synergies with their activation patterns are similar across directions (Fig. 8; Table 1).

Synergies 1 and 2, active at the swing-to-stance transition and early stance that mainly involve extensors were shared between forward and backward locomotion (Figs. 7, 8), serving to lower the hindlimbs for weight acceptance. Extensor half-centers or VC could control these synergies. The mostly similar relative activation of pure extensors (SOL, VL, BFA) during stance in both directions (synergies 1-3) appears to contribute to weight support. Although we did not find fully shared muscle synergies for hindlimb elevation, the relative activation of SRT, present in flexor synergies 4 and 5, weakly depended on locomotor direction and its mostly hip flexor action contributes to hindlimb elevation. Other muscles of synergies 4 and 5 , active at the stanceto-swing transition and swing (Figs. 7, 8), might receive common time-varying inputs from flexor half-centers. The proposed direction-dependent $\mathrm{HC}$ of hindlimb stepping may be generated by two-joint ankle extensors/knee flexors (LG, MG, and PLA) and knee flexors/hip extensors (ST and BFP) that propel the body during stance (synergies 3 and 4 ) and move the limb horizontally during swing (synergies 4 and 5; Figs. 7, 8). We explain the greater relative activity of these two-joint muscles during backward locomotion by their favorable mechanical advantage to accomplish task demands. Backward locomotion requires a combination of ankle extension-knee flexion moments and knee flexion-hip extension moments in the second half of stance phase, as opposed to ankle extension-knee extension and knee extension-hip flexion moments in forward locomotion. Early swing in backward locomotion requires a combination of knee flexion/hip extension moments, in contrast to a knee flexion/hip flexion moment during forward locomotion (Perell et al., 1993). Motoneurons of two-joint hindlimb muscles receive excitatory inputs during both flexion and extension phases (Perret and Cabelguen, 1980), presumably from corresponding half-centers, and from motion-dependent somatosensory feedback. The higher count of c-Fos positive interneurons for backward locomotion at L6-L7 segments (Merkulyeva et al., 2018), containing motoneurons and afferent projections of two-joint muscles (Hamm et al., 1985; VanderHorst and Holstege, 1997) supports their important role in the backward direction. Taken together, our analysis of muscle synergies is consistent with shared VC and distinct $\mathrm{HC}$ during forward and backward locomotion. We propose that shared components primarily control pure flexors and extensors while specialized mechanisms mainly control twojoint muscles for directional tuning. The proposed VC and HC mechanisms could be implemented in the spinal cord in two ways, as discussed above. The first by using fixed common synergy weights for both directions with direction-specific timevarying activation inputs affected by somatosensory feedback. The second by using a common time-varying input for both directions and direction-specific synergy weights shaped by somatosensory feedback and other signals.

It should be noted that our synergy analyses were conducted for individual cats with different numbers and sets of muscles, as well as for three cats combined but with a lower number of muscles, which could have affected the number, composition and activation patterns of obtained synergies.

\section{References}

Alluin O, Delivet-Mongrain H, Rossignol S (2015) Inducing hindlimb locomotor recovery in adult rat after complete thoracic spinal cord section 
using repeated treadmill training with perineal stimulation only. J Neurophysiol 114:1931-1946.

Andersson O, Grillner S (1983) Peripheral control of the cat's step cycle. II. Entrainment of the central pattern generators for locomotion by sinusoidal hip movements during fictive locomotion. Acta Physiol Scand 118:229-239.

Barbeau H, Julien C, Rossignol S (1987) The effects of clonidine and yohimbine on locomotion and cutaneous reflexes in the adult chronic spinal cat. Brain Res 437:83-96.

Bélanger M, Drew T, Provencher J, Rossignol S (1996) A comparison of treadmill locomotion in adult cats before and after spinal transection. J Neurophysiol 76:471-491.

Berkowitz A (2008) Physiology and morphology of shared and specialized spinal interneurons for locomotion and scratching. J Neurophysiol 99:2887-2901.

Buford JA, Smith JL (1990) Adaptive control for backward quadrupedal walking. II. Hindlimb muscle synergies. J Neurophysiol 64:756-766.

Buford JA, Smith JL (1993) Adaptive control for backward quadrupedal walking. III. Stumbling corrective reactions and cutaneous reflex sensitivity. J Neurophysiol 70:1102-1114.

Buford JA, Zernicke RF, Smith JL (1990) Adaptive control for backward quadrupedal walking. I. Posture and hindlimb kinematics. J Neurophysiol 64:745755.

Cappellini G, Sylos-Labini F, Maclellan MJ, Sacco A, Morelli D, Lacquaniti F, Ivanenko Y (2018) Backward walking highlights gait asymmetries in children with cerebral palsy. J Neurophysiol 119:1153-1165.

Cheung VC, d'Avella A, Tresch MC, Bizzi E (2005) Central and sensory contributions to the activation and organization of muscle synergies during natural motor behaviors. J Neurosci 25:6419-6434.

Choi JT, Bastian AJ (2007) Adaptation reveals independent control networks for human walking. Nat Neurosci 10:1055-1062.

Courtine G, Roy RR, Hodgson J, McKay H, Raven J, Zhong H, Yang H, Tuszynski MH, Edgerton VR (2005) Kinematic and EMG determinants in quadrupedal locomotion of a non-human primate (rhesus). J Neurophysiol 93:3127-3145.

Courtine G, Gerasimenko Y, van den Brand R, Yew A, Musienko P, Zhong H, Song B, Ao Y, Ichiyama RM, Lavrov I, Roy RR, Sofroniew MV, Edgerton VR (2009) Transformation of nonfunctional spinal circuits into functional states after the loss of brain input. Nat Neurosci 12:1333-1342.

Dambreville C, Labarre A, Thibaudier Y, Hurteau MF, Frigon A (2015) The spinal control of locomotion and step-to-step variability in left-right symmetry from slow to moderate speeds. J Neurophysiol 114:1119-1128.

de Guzman CP, Roy RR, Hodgson JA, Edgerton VR (1991) Coordination of motor pools controlling the ankle musculature in adult spinal cats during treadmill walking. Brain Res 555:202-214.

de Leon RD, Hodgson JA, Roy RR, Edgerton VR (1998) Locomotor capacity attributable to step training versus spontaneous recovery after spinalization in adult cats. J Neurophysiol 79:1329-1340.

Deliagina TG, Musienko PE, Zelenin PV (2019) Nervous mechanisms of locomotion in different directions. Curr Opin Physiol 8:7-13.

Desrochers E, Harnie J, Doelman A, Hurteau MF, Frigon A (2019) Spinal control of muscle synergies for adult mammalian locomotion. J Physiol 597:333-350.

Dietz V, Zijlstra W, Duysens J (1994) Human neuronal interlimb coordination during split-belt locomotion. Exp Brain Res 101:513-520.

Duysens J, Pearson KG (1980) Inhibition of flexor burst generation by loading ankle extensor muscles in walking cats. Brain Res 187:321-332.

Duysens J, Clarac F, Cruse H (2000) Load-regulating mechanisms in gait and posture: comparative aspects. Physiol Rev 80:83-133.

Duysens J, Tax AA, Murrer L, Dietz V (1996) Backward and forward walking use different patterns of phase-dependent modulation of cutaneous reflexes in humans. J Neurophysiol 76:301-310.

Finley JM, Bastian AJ, Gottschall JS (2013) Learning to be economical: the energy cost of walking tracks motor adaptation. J Physiol 591:1081-1095.

Forssberg H, Grillner S, Halbertsma J, Rossignol S (1980) The locomotion of the low spinal cat. II. Interlimb coordination. Acta Physiol Scand 108:283-295.

Frigon A (2009) Reconfiguration of the spinal interneuronal network during locomotion in vertebrates. J Neurophysiol 101:2201-2203.

Frigon A, Rossignol S (2008) Adaptive changes of the locomotor pattern and cutaneous reflexes during locomotion studied in the same cats before and after spinalization. J Physiol 586:2927-2945.
Frigon A, Hurteau MF, Thibaudier Y, Leblond H, Telonio A, D'Angelo G (2013) Split-belt walking alters the relationship between locomotor phases and cycle duration across speeds in intact and chronic spinalized adult cats. J Neurosci 33:8559-8566.

Frigon A, Thibaudier Y, Hurteau MF (2015) Modulation of forelimb and hindlimb muscle activity during quadrupedal tied-belt and split-belt locomotion in intact cats. Neuroscience 290:266-278.

Frigon A, Desrochers E, Thibaudier Y, Hurteau MF, Dambreville C (2017) Left-right coordination from simple to extreme conditions during splitbelt locomotion in the chronic spinal adult cat. J Physiol 595:341-361.

Goetz L, Piallat B, Thibaudier Y, Montigon O, David O, Chabardès S (2012) A non-human primate model of bipedal locomotion under restrained condition allowing gait studies and single unit brain recordings. J Neurosci Methods 204:306-317.

Gossard JP, Sirois J, Noué P, Côté MP, Ménard A, Leblond H, Frigon A (2011) Chapter 2-the spinal generation of phases and cycle duration. Prog Brain Res 188:15-29.

Grasso R, Bianchi L, Lacquaniti F (1998) Motor patterns for human gait: backward versus forward locomotion. J Neurophysiol 80:1868-1885.

Grillner S (1981) Control of locomotion in bipeds, tetrapods, and fish. In: Handbook of physiology, the nervous system, motor control (Brooks VB, ed), pp 1179-1236. Bethesda: Oxford University Press.

Grillner S (1985) Neurobiological bases of rhythmic motor acts in vertebrates. Science 228:143-149.

Grillner S, Rossignol S (1978) On the initiation of the swing phase of locomotion in chronic spinal cats. Brain Res 146:269-277.

Grillner S, El Manira A (2015) The intrinsic operation of the networks that make us locomote. Curr Opin Neurobiol 31:244-249.

Halbertsma JM (1983) The stride cycle of the cat: the modelling of locomotion by computerized analysis of automatic recordings. Acta Physiol Scand Suppl 521:1-75.

Hamm TM, Koehler W, Stuart DG, Vanden Noven S (1985) Partitioning of monosynaptic Ia excitatory post-synaptic potentials in the motor nucleus of the cat semimembranosus muscle. J Physiol 369:379-398.

Harnie J, Côté-Sarrazin C, Hurteau MF, Desrochers E, Doelman A, Amhis N, Frigon A (2018) The modulation of locomotor speed is maintained following partial denervation of ankle extensors in spinal cats. J Neurophysiol 120:1274-1285.

Harnie J, Doelman A, de VE, Audet J, Desrochers E, Gaudreault N, Frigon A (2019) The recovery of standing and locomotion after spinal cord injury does not require task-specific training. Elife 8 .

Hart CB, Giszter SF (2010) A neural basis for motor primitives in the spinal cord. J Neurosci 30:1322-1336.

Hofstoetter US, Knikou M, Guertin PA, Minassian K (2017) Probing the human spinal locomotor circuits by phasic step-induced feedback and by tonic electrical and pharmacological neuromodulation. Curr Pharm Des 23:1805-1820

Hoogkamer W, Meyns P, Duysens J (2014) Steps forward in understanding backward gait: from basic circuits to rehabilitation. Exerc Sport Sci Rev 42:23-29.

Ivanenko YP, Cappellini G, Poppele RE, Lacquaniti F (2008) Spatiotemporal organization of alpha-motoneuron activity in the human spinal cord during different gaits and gait transitions. Eur J Neurosci 27:3351-3368.

Jensen L, Prokop T, Dietz V (1998) Adaptational effects during human splitbelt walking: influence of afferent input. Exp Brain Res 118:126-130.

Kiehn O (2011) Development and functional organization of spinal locomotor circuits. Curr Opin Neurobiol 21:100-109.

Kiehn O (2016) Decoding the organization of spinal circuits that control locomotion. Nat Rev Neurosci 17:224-238.

Kilkenny C, Browne WJ, Cuthill IC, Emerson M, Altman DG (2010) Improving bioscience research reporting: the ARRIVE guidelines for reporting animal research. PLoS Biol 8:e1000412.

Klishko AN, Farrell BJ, Beloozerova IN, Latash ML, Prilutsky BI (2014) Stabilization of cat paw trajectory during locomotion. J Neurophysiol 112:1376-1391.

Kuczynski V, Telonio A, Thibaudier Y, Hurteau MF, Dambreville C, Desrochers E, Doelman A, Ross T, Frigon A (2017) Lack of adaptation during prolonged split-belt locomotion in the intact and spinal cat. J Physiol 595:5987-6006.

Kulagin AS, Shik ML (1970) [Interaction of symmetric extremities during controlled locomotion]. Biofizika 15:164-170. 
Lamb T, Yang JF (2000) Could different directions of infant stepping be controlled by the same locomotor central pattern generator? J Neurophysiol 83:2814-2824.

Leblond H, L'Esperance M, Orsal D, Rossignol S (2003) Treadmill locomotion in the intact and spinal mouse. J Neurosci 23:11411-11419.

Lee DD, Seung HS (1999) Learning the parts of objects by non-negative matrix factorization. Nature 401:788-791.

Li WC, Sautois B, Roberts A, Soffe SR (2007) Reconfiguration of a vertebrate motor network: specific neuron recruitment and context-dependent synaptic plasticity. J Neurosci 27:12267-12276.

Liao JC, Fetcho JR (2008) Shared versus specialized glycinergic spinal interneurons in axial motor circuits of larval zebrafish. J Neurosci 28:1298212992.

Lovely RG, Gregor RJ, Roy RR, Edgerton VR (1990) Weight-bearing hindlimb stepping in treadmill-exercised adult spinal cats. Brain Res 514:206218.

Mahaki M, De Sá E, Souza GS, Mimar R, Vieira MF (2017) The comparison of ground reaction forces and lower limb muscles correlation and activation time delay between forward and backward walking. Gait Posture 58:380-385.

Matsushima T, Grillner S (1992) Neural mechanisms of intersegmental coordination in lamprey: local excitability changes modify the phase coupling along the spinal cord. J Neurophysiol 67:373-388.

McCrea DA, Rybak IA (2008) Organization of mammalian locomotor rhythm and pattern generation. Brain Res Rev 57:134-146.

Merkulyeva N, Veshchitskii A, Gorsky O, Pavlova N, Zelenin PV, Gerasimenko Y, Deliagina TG, Musienko P (2018) Distribution of spinal neuronal networks controlling forward and backward locomotion. J Neurosci 38:4695-4707.

Merlet AN, Harnie J, Macovei M, Doelman A, Gaudreault N, Frigon A (2020) Mechanically stimulating the lumbar region inhibits locomotorlike activity and increases the gain of cutaneous reflexes from the paws in spinal cats. J Neurophysiol 123:1026-1041.

Musienko PE, Bogacheva IN, Gerasimenko YP (2007) Significance of peripheral feedback in the generation of stepping movements during epidural stimulation of the spinal cord. Neurosci Behav Physiol 37:181-190.

Musienko PE, Zelenin PV, Lyalka VF, Gerasimenko YP, Orlovsky GN, Deliagina TG (2012) Spinal and supraspinal control of the direction of stepping during locomotion. J Neurosci 32:17442-17453.

Pearson KG (2008) Role of sensory feedback in the control of stance duration in walking cats. Brain Res Rev 57:222-227.

Perell KL, Gregor RJ, Buford JA, Smith JL (1993) Adaptive control for backward quadrupedal walking. IV. Hindlimb kinetics during stance and swing. J Neurophysiol 70:2226-2240.

Perret C, Cabelguen JM (1980) Main characteristics of the hindlimb locomotor cycle in the decorticate cat with special reference to bifunctional muscles. Brain Res 187:333-352.
Pratt CA, Buford JA, Smith JL (1996) Adaptive control for backward quadrupedal walking V. Mutable activation of bifunctional thigh muscles. J Neurophysiol 75:832-842.

Prokop T, Berger W, Zijlstra W, Dietz V (1995) Adaptational and learning processes during human split-belt locomotion: interaction between central mechanisms and afferent input. Exp Brain Res 106:449-456.

Reisman DS, Block HJ, Bastian AJ (2005) Interlimb coordination during locomotion: what can be adapted and stored? J Neurophysiol 94:24032415.

Robinson GA, Goldberger ME (1986) The development and recovery of motor function in spinal cats. I. The infant lesion effect. Exp Brain Res 62:373-386.

Rossignol S, Frigon A (2011) Recovery of locomotion after spinal cord injury: some facts and mechanisms. Annu Rev Neurosci 34:413-440.

Rossignol S, Chau C, Brustein E, Giroux N, Bouyer L, Barbeau H, Reader TA (1998) Pharmacological activation and modulation of the central pattern generator for locomotion in the cat. Ann NY Acad Sci 860:346-359.

Rossignol S, Dubuc R, Gossard JP (2006) Dynamic sensorimotor interactions in locomotion. Physiol Rev 86:89-154.

Takei T, Confais J, Tomatsu S, Oya T, Seki K (2017) Neural basis for hand muscle synergies in the primate spinal cord. Proc Natl Acad Sci USA 114:8643-8648

Thibaudier Y, Frigon A (2014) Spatiotemporal control of interlimb coordination during transverse split-belt locomotion with 1:1 or 2:1 coupling patterns in intact adult cats. J Neurophysiol 112:2006-2018.

Thorstensson A (1986) How is the normal locomotor program modified to produce backward walking? Exp Brain Res 61:664-668.

Ting LH, McKay JL (2007) Neuromechanics of muscle synergies for posture and movement. Curr Opin Neurobiol 17:622-628.

Trank TV, Smith JL (1996) Adaptive control for backward quadrupedal walking VI. metatarsophalangeal joint dynamics and motor patterns of digit muscles. J Neurophysiol 75:678-679.

Tresch MC, Jarc A (2009) The case for and against muscle synergies. Curr Opin Neurobiol 19:601-607.

VanderHorst VG, Holstege G (1997) Organization of lumbosacral motoneuronal cell groups innervating hindlimb, pelvic floor, and axial muscles in the cat. J Comp Neurol 382:46-76.

Viviani P, Figliozzi F, Campione GC, Lacquaniti F (2011) Detecting temporal reversals in human locomotion. Exp Brain Res 214:93-103.

Winter DA, Pluck N, Yang JF (1989) Backward walking: a simple reversal of forward walking? J Mot Behav 21:291-305.

Yang Q, Logan D, Giszter SF (2019) Motor primitives are determined in early development and are then robustly conserved into adulthood. Proc Natl Acad Sci USA 116:12025-12034.

Zelenin PV, Deliagina TG, Orlovsky GN, Karayannidou A, Stout EE, Sirota MG, Beloozerova IN (2011) Activity of motor cortex neurons during backward locomotion. J Neurophysiol 105:2698-2714. 